\title{
LES SUBMERSIONS MARINES EN MANCHE ORIENTALE : APPROCHE INDUCTIVE ET NATURALISTE POUR LA CARACTÉRISATION DES FACTEURS RESPONSABLES DES INONDATIONS PAR LA MER
}

\author{
Pauline LETORTU, Stéphane COSTA et Olivier CANTAT \\ LETG-Caen GEOPHEN \\ Laboratoire Géographie Physique et Environnement \\ Université de Caen Basse-Normandie, UMR LETG 6554 CNRS \\ Esplanade de la Paix, BP 5186 \\ 14032 Caen Cedex 5, France \\ pauline.letortu@unicaen.fr,stephane.costa@unicaen.fr,olivier.cantat@unicaen.fr
}

\begin{abstract}
Résumé
Après le passage de la tempête Xynthia fin février 2010, dont la gravité des inondations par la mer sur le littoral atlantique et en Manche a été exceptionnelle (en France, 47 morts et près de 2 milliards d'euros de dommages), l'étude des phénomènes météo-marins à l'origine de ces franchissements et leur prévision/prévention a connu un net regain d'intérêt. Cet article porte sur les côtes basses urbanisées du littoral normano-picard qui cumule de nombreux facteurs de prédisposition (ouverture aux flux d'ouest perturbés, crise sédimentaire des plages et faible altimétrie des vallées). Pour ces trois principales raisons, ces secteurs revêtent un intérêt tout particulier pour l'analyse du risque d'inondation par la mer. L'objectif du travail est double : d'une part, présenter l'inventaire des inondations (sur la période 1949-2010) et détecter une éventuelle évolution de la fréquence et de l'intensité de ces événements, en relation possible avec le changement climatique actuel ; d'autre part, caractériser les divers paramètres météo-marins et morphologiques des plages aboutissant aux inondations par la mer. Au total, le croisement de la base de données « submersion » et des facteurs météo-marins confirme le rôle essentiel de la concomitance de conditions bien connues (fort vent d'afflux et haut niveau marin), ici renforcées par le passage d'un front froid au moment de la pleine mer. Mais quelques cas ne répondant pas à cette logique nous ont conduits à reprendre pas à pas la chaîne des facteurs explicatifs en adoptant une démarche inductive et naturaliste. Il s'agit de mettre en évidence l'action d'autres facteurs responsables des franchissements jusqu'à présent mal appréhendés. Ce nouveau regard, fondé sur l'observation de terrain et l'emboîtement des échelles spatiales et temporelles, a notamment permis de mettre en évidence le «temps de préparation » de la plage (abaissement de son profil) par des conditions météo-marines non nécessairement extrêmes, antérieures à l'inondation.
\end{abstract}

Mots-clés : submersions marines, Manche orientale, météorologie, profil de plage, prévision/prévention.

\section{Abstract \\ Coastal flooding in the French coast of the eastern English Channel: inductive and naturalistic approaches for the characterization of factors ending in flooding}

Xynthia storm, in February 2010, has left its mark on the French coast of Atlantic Ocean and the English Channel because of its severity (47 people died and about $€ 2$ billion in damages). Consequently, the current context gives strong interest in studies about these meteorological and marine phenomena and their weather forecasting/prevention. In this paper, we studied French low coastal areas of the eastern English Channel (Normandy and Picardy coasts) because of their sensitivity to coastal flooding risk. Because of cumulative factors (exposure to strong West wind, beach sedimentary crisis, low altimetry of valleys), the French coast of the eastern English Channel is particularly interesting for analysis of this natural risk. This work has a double objective. The first objective is the presentation of our coastal flooding inventory (from 1949 to 2010) and the detection of a possible evolution about frequency and intensity of coastal flooding events perhaps linked to current climate change. The second objective is the characterization of the different meteorological, marine, and morphological parameters that end in these destructive phenomena. In matching these meteorological and marine factors with the past events inventory, we have identified the dominance of the concomitancy of well-known conditions (strong wind perpendicular to coast and high tide level), reinforced here by the passage of an atmospheric cold front during high tide. But some cases do not follow this logic. To understand them, we have followed step by step the explanation process in adopting inductive and naturalistic approaches. The aim is to

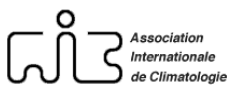


highlight other factors that are involved in the process of coastal flooding. This new point of view is based on field observation and the use of different spatial and temporal scales and enables to emphasize "the preparation time" of the beach (lowering of its profile) by meteorological and marine conditions (not necessarily extreme) before the coastal flooding event.

Keywords: coastal flooding, the eastern English Channel, meteorology, beach profile, weather forecasting/prevention.

\section{Introduction}

De par leurs conséquences environnementales et sociétales, les submersions marines ont toujours suscité l'intérêt des chercheurs ${ }^{1}$. La submersion marine, souvent due aux tempêtes d'où le terme fréquemment employé de «submersion de tempête » (Galli et Hontarrède, 2001) - est le résultat d'une conjonction de facteurs bien connus: une faible pression atmosphérique, un fort vent d'afflux (ces deux facteurs peuvent déterminer une surcote ou " onde de tempête ») au moment d'une pleine mer de vive-eau. Mais des tempêtes peuvent être sans submersion et il peut se produire des submersions sans véritable tempête. Pour ces raisons, nous préférons le terme de "submersion marine " car il n'induit pas un lien de causalité dans l'origine du phénomène. Par ailleurs, le terme de "submersion marine » a été choisi pour le futur PPRL ${ }^{2}$. La grande majorité des submersions marines sur le secteur étudié correspondent à un franchissement et un rabattement des vagues sur les ouvrages.

Le littoral français de la Manche orientale, compris entre Le Havre (Seine-Maritime) et Cayeux-sur-Mer (Somme), est sensible aux inondations par la mer en raison de son ouverture aux flux d'ouest perturbés et de ses caractéristiques morphologiques et altimétriques. Entre les célèbres falaises de craie qui identifient ce littoral, telle Etretat, l'urbanisation importante des côtes basses (Fécamp, Saint-Valéry-en-Caux, Dieppe, Le Tréport...) accentue la vulnérabilité de ces espaces parfois situés à plus d'un mètre en-dessous des pleines mers de vive-eau. Cette vulnérabilité explique l'attention particulière et croissante portée sur ces territoires ces dernières années (Caspar, 1988, 1990 ; Caspar et Poullain, 1996 ; Caspar et al., 2007, 2010 ; Costa, 1997, 2005 ; Costa et al., 2002, 2004 ; Costa et Delahaye, 2005 ; Elineau et al., 2011).

Dans cet article, il s'agira de prolonger temporellement ces études (notamment celle de Stéphane Costa en 1997) grâce à un recensement des inondations par la mer qui sont apparues entre 1949 et 2010. Cet inventaire permettra d'apporter des éléments de réponse quant à

\footnotetext{
${ }^{1}$ Citons quelques exemples en mer Baltique (Herrmann et Planchon, 1998 ; Woth et al., 2006 ; Tõnisson et al., 2008), en mer du Nord (Vasseur et Héquette, 2000 ; Woth et al., 2006), en Manche (Caspar, 1988, 1990 ; Caspar et Poullain, 1996 ; Caspar et al., 2007, 2010 ; Costa, 1997, 2005 ; Tonnerre, 2001 ; Costa et al., 2002, 2004, 2005 ; Pirazzoli et al., 2006 ; Elineau et al., 2011), en Atlantique (Tonnerre, 2001 ; Hallégouët et Hénaff, 2006 ; Le Cornec et Schoorens, 2007 ; Suanez et al., 2007 ; Cariolet et Suanez, 2009 ; Peeters et al., 2009 ; Cariolet et al., 2010, Cariolet, 2011) et en Méditerranée (Bruzzi, 1998 ; Anselme et al., 2008 ; Suanez, 2009).

${ }^{2}$ Les submersions marines sont des inondations temporaires de la zone côtière par la mer dans des conditions météorologiques et océaniques défavorables (basses pressions atmosphériques et fort vent d'afflux agissant, pour les mers à marée, lors d'une pleine mer de vive-eau); elles peuvent durer de quelques heures à quelques jours. Trois modes de submersion marine sont distingués (Perherin et al., 2012) :

1) submersion par débordement, lorsque le niveau marin est supérieur à la cote de crête des ouvrages ou du terrain naturel,

2) submersion par franchissement de paquets de mer liés aux vagues, lorsqu'après déferlement de la houle, les paquets de mer dépassent la cote de crête des ouvrages ou du terrain naturel,

3) submersion par rupture du système de protection, lorsque les terrains situés en arrière sont en dessous du niveau marin : défaillance d'un ouvrage de protection ou formation de brèche dans un cordon naturel suite à l'attaque de la houle (énergie libérée lors du déferlement), au mauvais entretien d'un ouvrage, à une érosion chronique intensive, au phénomène de sur-verse, à un déséquilibre sédimentaire du cordon naturel, etc.
} 
l'évolution de la fréquence des submersions marines depuis 60 ans ainsi que sur la définition des paramètres météo-marins et morphologiques des plages favorables aux franchissements par la mer.

La méthode de travail adoptée dans cette recherche vise à être reproductible et transposable à d'autres espaces et s'appuie donc sur des données facilement accessibles (gratuites et d'usage courant). La démarche proposée est inductive et naturaliste : en se fondant sur un inventaire historique des submersions marines, notre analyse part de la transformation en dommages concrets d'un système à fort risque. Par la suite, nous en cherchons les causes, notamment atmosphériques (par exemple la force et la direction du vent) mais pas uniquement. En effet, si la présence de conditions tempétueuses est la plupart du temps incriminée, ces conditions atmosphériques n'expliquent pas la totalité des submersions avérées. Cela suppose l'intervention d'autres forçages favorables aux inondations par la mer se surimposant à ceux ordinairement observés.

Dans un premier temps, les caractéristiques géographiques et climatiques des côtes normano-picardes seront présentées dans la mesure où elles conditionnent grandement le fonctionnement du phénomène «submersion marine ». Dans un deuxième temps, la méthodologie d'inventaire des inondations sera décrite et une analyse de leur fréquence et intensité sera proposée. La troisième partie sera consacrée plus directement à l'étude des conditions météo-marines menant au franchissement. La dernière partie s'intéressera plus précisément aux conditions météo-marines préalables aux submersions et notamment leur rôle potentiellement négatif sur les caractéristiques morpho-sédimentaires des plages de galets haut-normandes et picardes. Une attention toute particulière sera ainsi portée aux variations du profil des plages dont l'importance semble essentielle lorsque les conditions météomarines ne sont pas optimales pour générer des submersions.

\section{Présentation géographique et climatique de la Manche orientale}

La Manche est une mer épicontinentale et intracontinentale située entre le sud du Royaume-Uni et la partie nord occidentale de la France (figure 1). Caractérisée par une faible profondeur (86 mètres en moyenne et rarement plus de 50 mètres dans sa partie orientale), la Manche est sous l'influence de forts marnages ( 8 mètres à Dieppe) et d'une fréquence importante de vents forts à dominante d'Ouest.

Le secteur d'étude s'étend entre la baie de Seine et la baie de Somme, et plus précisément du Havre, en Haute-Normandie, à Cayeux-sur-Mer, en Picardie (figure 2).

\subsection{Les principaux caractères topographiques et morphologiques de la côte normano- picarde}

Le linéaire côtier étudié $(145 \mathrm{~km})$, situé à la terminaison nord-occidentale du Bassin Parisien, est caractérisé par des côtes à falaises d'altitude moyenne de 70 mètres. Ces abrupts sont taillés pour l'essentiel dans des craies du Crétacé supérieur plus ou moins riches en lits de silex. Ces falaises sont localement entrecoupées par des vallées basses urbanisées dont le plancher est parfois inférieur d'un mètre aux pleines mers de vive-eau, ce qui en fait des secteurs à risques majeurs vis-à-vis des submersions marines. A titre d'illustration, ce contexte classique en Haute-Normandie est illustré par le cliché de la figure 3.

Au débouché de ces vallées se trouve un cordon de galets adossé à un perré. Selon la classification de Jennings et Shulmeister (2002), les plages de galets haut-normandes sont dites « composites ». Elles sont constituées d'un cordon de galets pentu d'aspect réfléchissant (inclinaison de 5 à $15 \%$ ), composé d'un mélange de galets, de graviers et de sables. Au pied

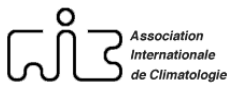


de ce cordon, nous retrouvons fréquemment un estran sableux d'environ 200 mètres de large, recouvrant un platier rocheux. Cet espace intertidal est caractérisé par une faible pente (inclinaison de 0,5 à $1,5 \%$ ), donnant à ce segment de plage un aspect fortement dissipatif.

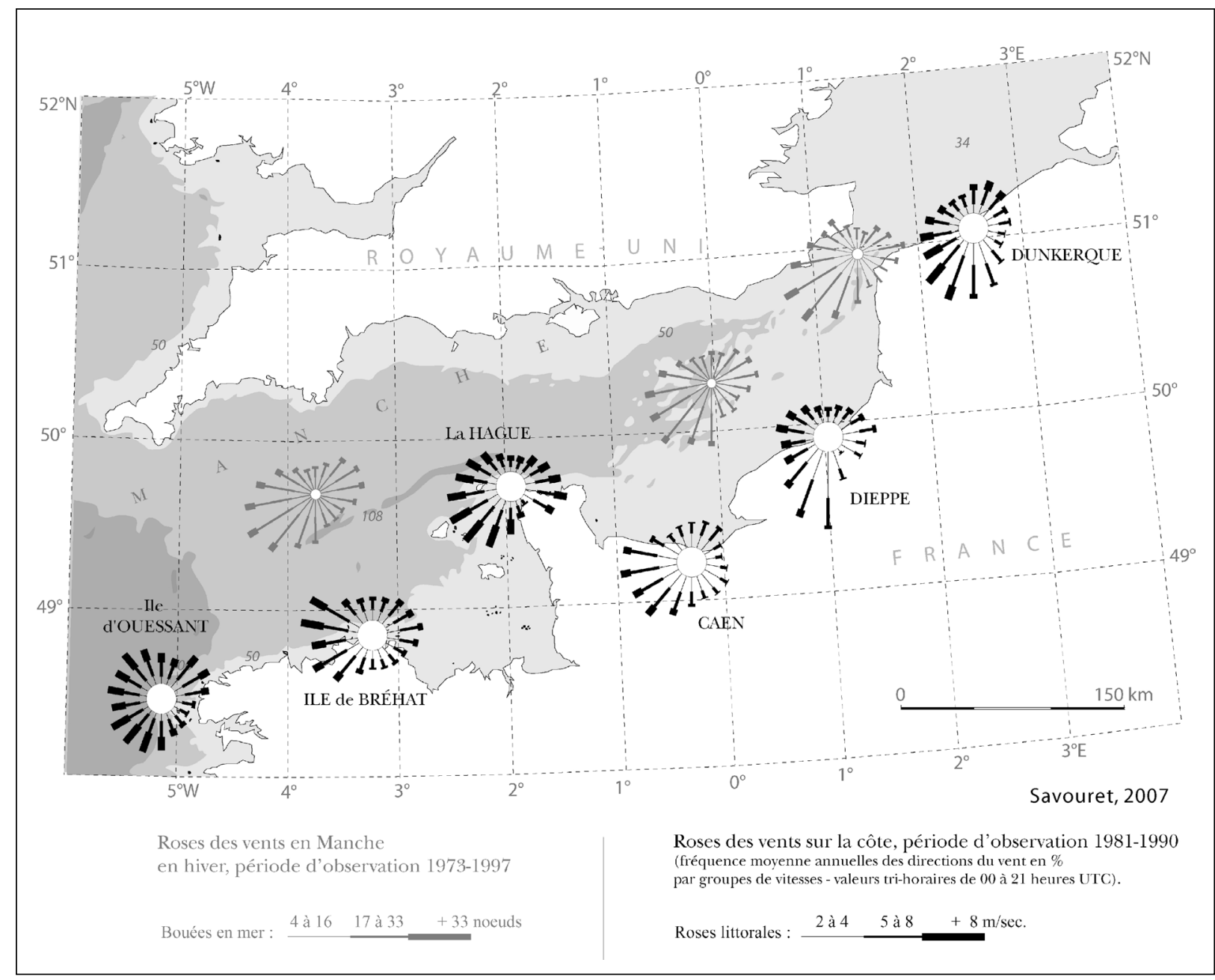

Figure 1 : Bathymétrie et roses des vents en Manche, en mer (hiver) et sur le littoral (Savouret, 2007). Bathymetry and wind roses in the English Channel, at sea (winter) and at the seaside (Savouret, 2007).

Les zones basses sont donc protégées à leur débouché par un cordon de galets dont la largeur moyenne est de 40 mètres et peut atteindre jusqu'à 60 mètres. La base de ces cordons se situe à environ 3 mètres d'altitude (cote marine) et leur crête dépasse souvent les plus hautes mers de 2 à 3 mètres (12,1 à 13,1 m à Dieppe en cote marine). La présence d'ouvrages transversaux (épis) explique que le volume de galets au droit des côtes basses urbanisées est plus important que celui se trouvant au pied des falaises (15 à 20 mètres) (figure 3). Lors de forts coups de vent d'afflux agissant lors d'une pleine mer de vive-eau, ce sont ces ouvrages qui sont franchis par la mer.

\subsection{Les principaux caractères météo-climatiques de la côte normano-picarde}

De part et d'autre du $50^{\text {ème }}$ parallèle nord, la Manche orientale appartient à l'aire du climat «tempéré de façade ouest» (Flohn, 1968), domaine caractérisé par de faibles contrastes thermiques et des précipitations qui se répartissent assez régulièrement sur l'année. Ce secteur, qualifié d' "éventé » par Gisèle Escourrou (1978), est de fait caractérisé par une grande fréquence des vents forts à très forts (figure 1). Cet élément constitue un paramètre météorologique majeur puisqu'il génère la houle et les vagues. Les tempêtes atlantiques 
hivernales circulent généralement à des latitudes un peu plus élevées, entre Irlande et Écosse (Trzpit, 1977 et 1990) mais il n'est cependant pas rare que certaines s'engouffrent dans le goulet d'étranglement constitué par la Manche, occasionnant alors de sérieux dommages tant matériels qu'humains.

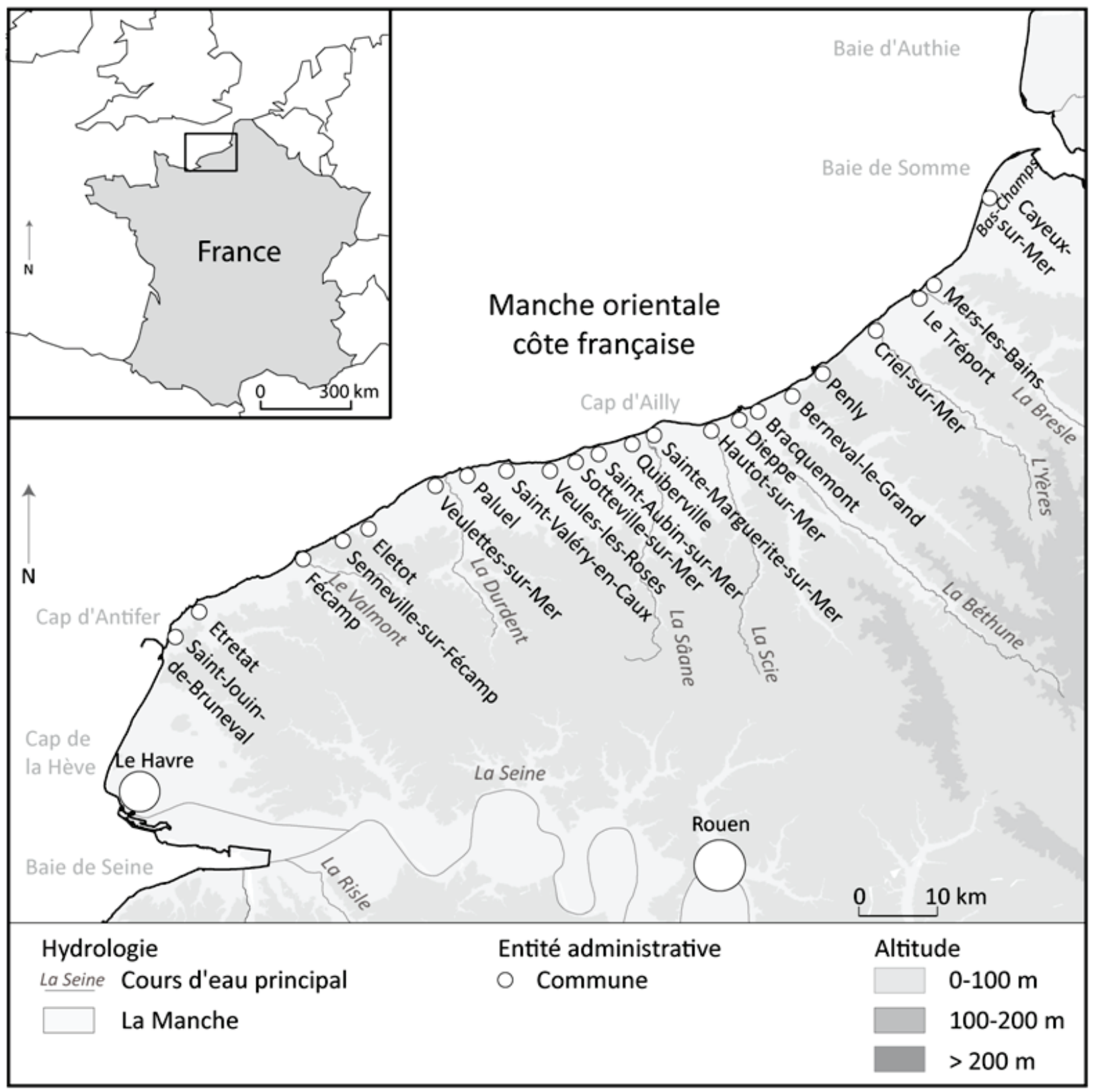

Figure 2 : Localisation du secteur d'étude. Location of the study area.

Dans une étude appliquée aux risques de submersion, notons que la diversité d'exposition sur le littoral normano-picard entraîne des contrastes sensibles dans la force et la direction du vent (figure 4), expliquant en partie la diversité spatiale des dégâts occasionnés. Les sites dégagés de Dinard, du cap de La Hague et de Bernières-sur-Mer offrent une bonne représentativité climatologique pour cette approche d'échelle subrégionale (Cantat et al., 2009). La principale opposition apparaît entre la côte occidentale du nord Cotentin, surexposée aux flux rapides de composantes ouest (station de référence : La Hague), et la baie de Seine, en position d'abri pour ces mêmes flux car en partie protégée entre le Cotentin et l'avancée du Pays de Caux (station de référence : Bernières-sur-Mer). 


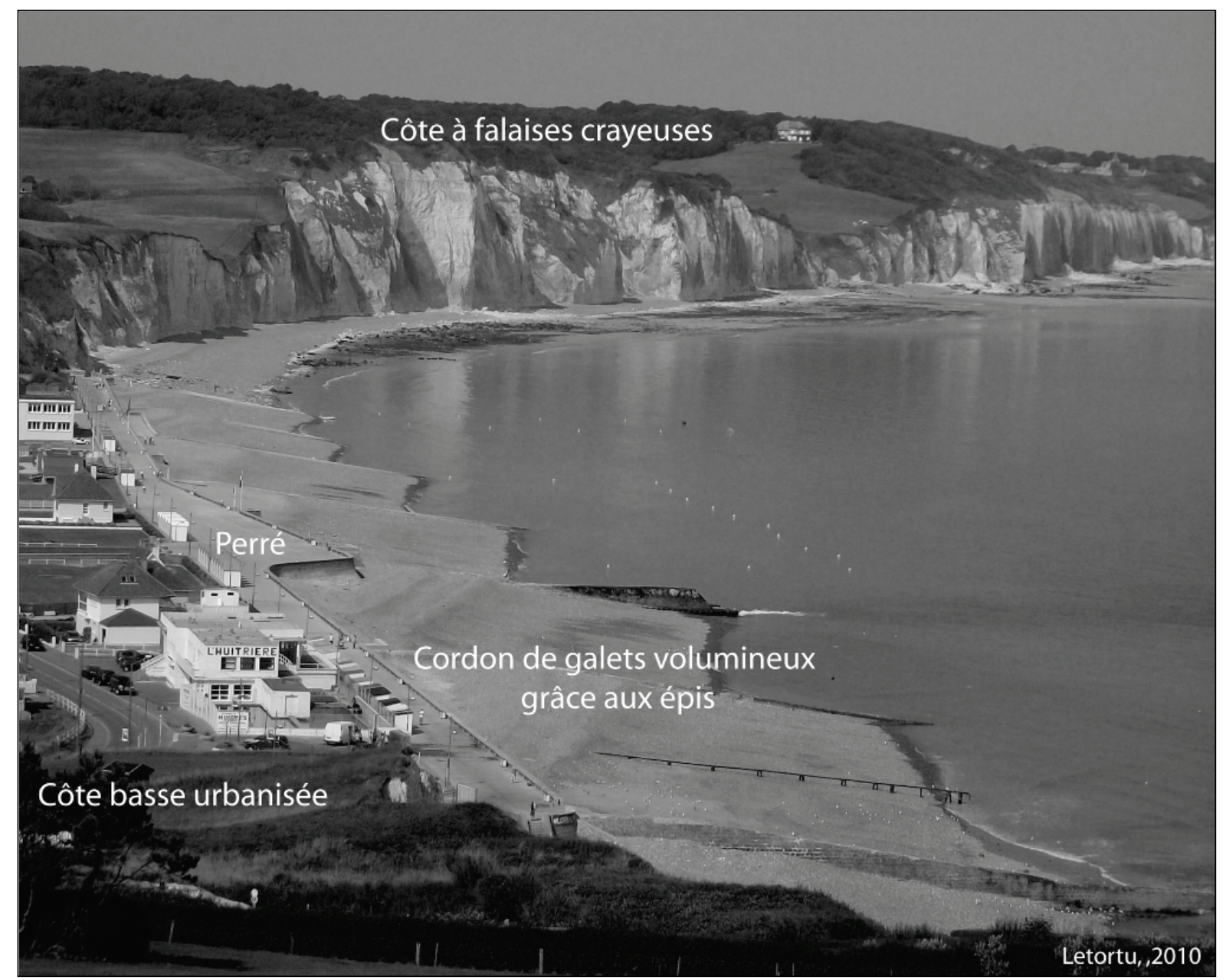

Figure 3 : Côte basse de Hautot-sur-Mer protégée par le cordon de galets et les épis. Low coastal area of Hautot-sur-Mer protected by the gravel beach and the beach groynes.

A une échelle plus fine, les effets de site perturbent l'écoulement de l'air, induisant parfois des déformations notables de la rose des vents (Leroy et Lafaysse, 1993 ; Leroy, 1999). Ainsi, le contexte stationnel du sémaphore de la pointe du Roc (présence de falaises), situé au nord de la baie du Mont-Saint-Michel, dévie les vents le long du littoral selon un axe privilégié nord-nord-ouest / sud-sud-est et celui de Dieppe atténue pour la même raison la force des violentes rafales provenant du quadrant sud. Ces éléments seront repris plus spécifiquement lors de l'analyse des conditions météo-marines menant au franchissement en Manche orientale (voir partie 3).

Conformément aux données de vent, les houles les plus fréquentes et les plus fortes apparaissent entre octobre et mars et sont, par ordre d'importance, d'ouest à sud-ouest, puis de nord-ouest, alors que les houles de nord et de nord-est sont bien évidemment plus faibles (Allen et Delannoy, 1990). Pour la station de Dieppe, en considérant la canalisation des flux et l'orientation de la côte, la période la plus sensible aux afflux d'eau sur le littoral est comprise entre novembre et février et largement dominée par les flux provenant du quadrant ouvert à l'ouest (figure 5).

La période propice aux fortes houles correspond à celle des tempêtes, mais la géométrie de la Manche génère des fetchs réduits pour quasiment toutes les directions de vent n'induisant alors qu'une agitation de type «mer du vent». Cependant, les hauteurs de vagues peuvent atteindre jusqu'à 4,3 mètres (période de retour annuelle du $\mathrm{H}_{1 / 3}$ à Dieppe) (Allen et Delannoy, 1990). Un seul fuseau étroit bénéficie de houles du large : le plein ouest. 


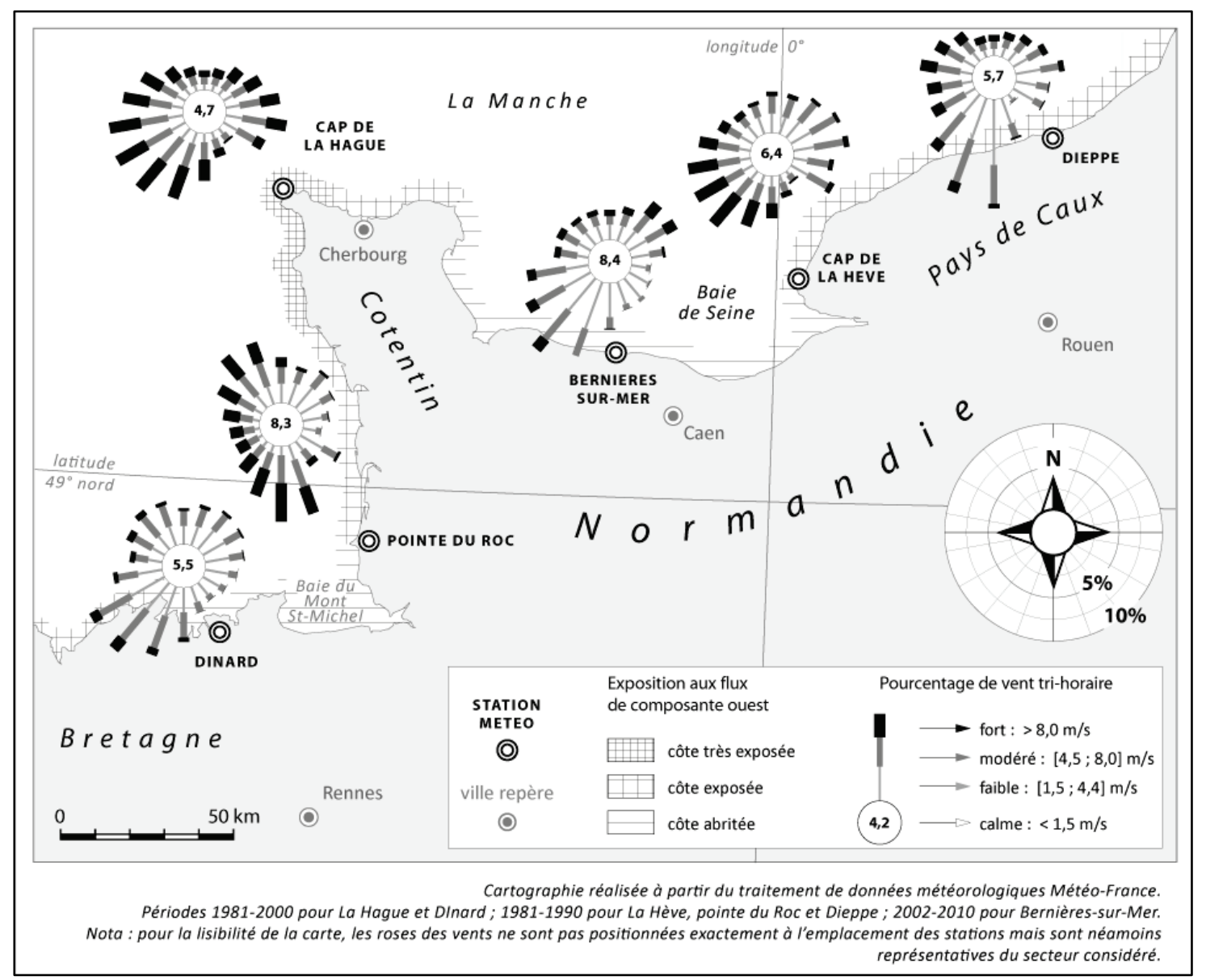

Figure 4 : Diversité régionale du vent tri-horaire sur la période 1981-2010 et exposition aux flux de composante ouest sur les côtes normano-picardes. Regional diversity of the three hourly wind (1981-2010) and the exposure of flow of West component on Normandy and Picardy coasts.

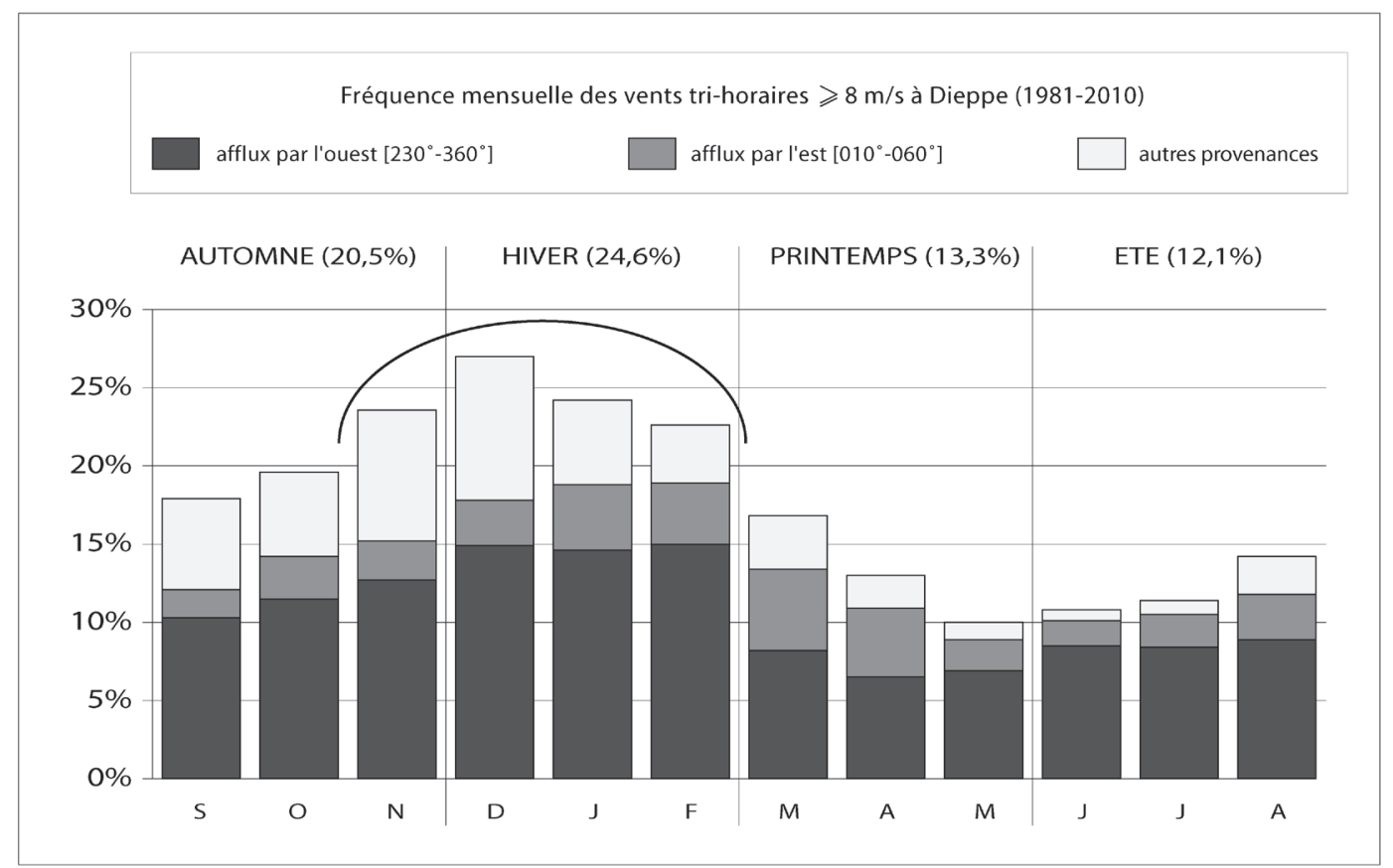

Figure 5 : Fréquence et saisonnalité des vents forts tri-horaires $(\geq 8 \mathrm{~m} / \mathrm{s})$ générateurs de fortes houles provenant de l'ouest ou de l'est à Dieppe sur la période 1981-2010. Frequency and seasonality of the fast three hourly winds $(\geq 8 \mathrm{~m} / \mathrm{s})$ generators of strong West or East swells in Dieppe over the period 1981-2010. 
En résumé, la Manche orientale française constitue un espace où la conjonction des facteurs géographiques et dynamiques prédispose tout particulièrement les sites de basses vallées aux submersions marines étant donné leur exposition directe aux flux les plus forts et aux fetchs les plus longs.

\section{Approche historique des submersions marines en Manche orientale}

La recherche sur les risques dits naturels nécessite de disséquer les forçages associés sur le long terme.

\subsection{L'inventaire des submersions marines en Manche orientale}

Le travail historique d'inventaire des inondations par la mer a été effectué sur 62 années consécutives (1949-2010) à partir de sources diverses : journaux locaux, base de données du MEDDE (Ministère de l'Ecologie, du Développement Durable et de l'Energie), thèse de Stéphane Costa (1997), archives du C.E.T.E. de Rouen, archives communales, articles scientifiques... Chacune de ces sources présente des avantages et des inconvénients. Les dates trouvées ont été croisées avec la base de données marégraphique du SHOM, pour identifier les cas suspects (cote en-dessous des pleines mers de vive-eau).

Notre source d'information principale provient de la presse locale. Deux journaux ont été particulièrement analysés : Les informations dieppoises et Liberté de Normandie. Les atouts de la presse locale sont l'immédiateté des renseignements et la bonne vision de la submersion telle que la perçoivent les populations locales au moment même de l'événement. On peut ainsi obtenir des données qualitatives et quantitatives sur les hauteurs d'eau atteintes. Souvent, les autres secteurs touchés sont évoqués ainsi que le dernier événement passé de même intensité (Agasse, 2005). Quant aux limites, les effets de contexte sont évidents avec notamment une augmentation du nombre d'articles consacrés aux risques naturels à partir des décennies 1980. Ce phénomène de résonance journalistique est très souvent accru après des phénomènes majeurs, comme Xynthia en février 2010. A contrario, en contexte d'actualités jugées «plus importantes » par l'équipe éditoriale, certaines inondations par la mer, pourtant d'intensité sévère, peuvent presque passer inaperçues. Ce phénomène de banalisation journalistique s'est produit lors de la submersion de la nuit du 10 au 11 mars 2008, en période d'élections municipales. Une autre limite observée à l'utilisation des journaux est parfois l'absence de précision sur la date et la localisation des événements : "la semaine dernière », « sur la côte »... Ces indications permettent, certes d'inventorier les faits mais n'offrent pas la possibilité d'analyser en détail l'événement (lieu, sévérité de l'événement...). Dans un souci d'exhaustivité, toutes les sources d'information disponibles ont été utilisées. Ce travail méthodique de recoupement des informations de sources diverses nous a permis de garantir la fiabilité et la représentativité géographique de l'inventaire. Au total, de 1949 à 2010, 78 submersions marines ont été recensées sur les 145 kilomètres de côte s'étendant du Havre à Cayeux-sur-Mer. Ces inondations par la mer ont eu des conséquences variées sur le littoral, allant du simple franchissement localisé des ouvrages de défense à l'envahissement de l'intérieur de la commune.

\subsection{La classification des submersions marines}

Plusieurs classifications de la gravité des submersions marines existent (Pirazzoli et al., 2007 ; SIRACEDPC, 2008). Rémi Caspar (Météo-France) a proposé trois degrés de gravité pour la rédaction du Plan ORSEC, sous l'égide de la Préfecture de Seine-Maritime (SIRACEDPC, 2008). "Une submersion 'faible' correspond à un envahissement par la mer de l'ensemble d'une plage et de la digue promenade (perré), ou encore un dépassement faible 
de quais portuaires. Une submersion 'modérée' fait pénétrer les eaux sur la voie publique la plus proche d'un perré ou d'un quai portuaire. Une submersion dite 'sévère' pousse les eaux à l'intérieur d'une commune et y provoque des dégâts relevant de la 'catastrophe naturelle'». Cette classification d'échelle départementale a été choisie pour notre étude ${ }^{3}$, en s'appuyant notamment sur les descriptions et les photographies fournies par la presse locale (figure 6).

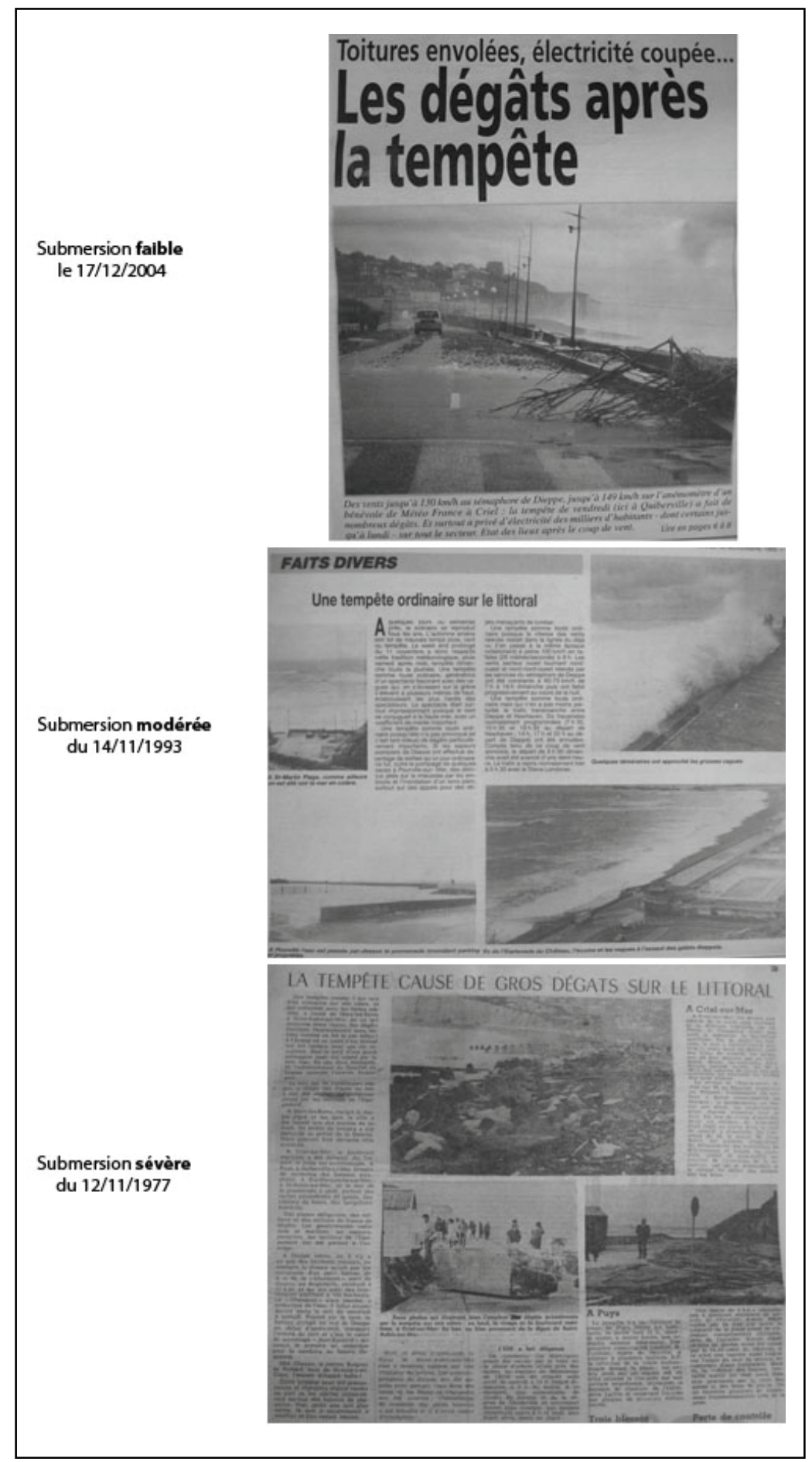

Figure 6: Degrés de gravité des submersions marines (coupures d'articles Les informations dieppoises). Severity degrees of coastal flooding events (from the newspaper Les informations dieppoises).

\footnotetext{
${ }^{3}$ En raison de la subjectivité et de la résolution spatiale de cette classification, une échelle de gravité de l'inondation pour chaque site serait nécessaire pour affiner l'analyse et fera l'objet d'un travail futur.
} 


\subsection{L'évolution des submersions marines entre 1949 et 2010 en Manche orientale}

Sur les 78 submersions marines recensées, 12 sont de gravité faible, 12 de gravité modérée et 48 de gravité sévère ; 6 cas ne peuvent être déterminés faute de description (figure 7).

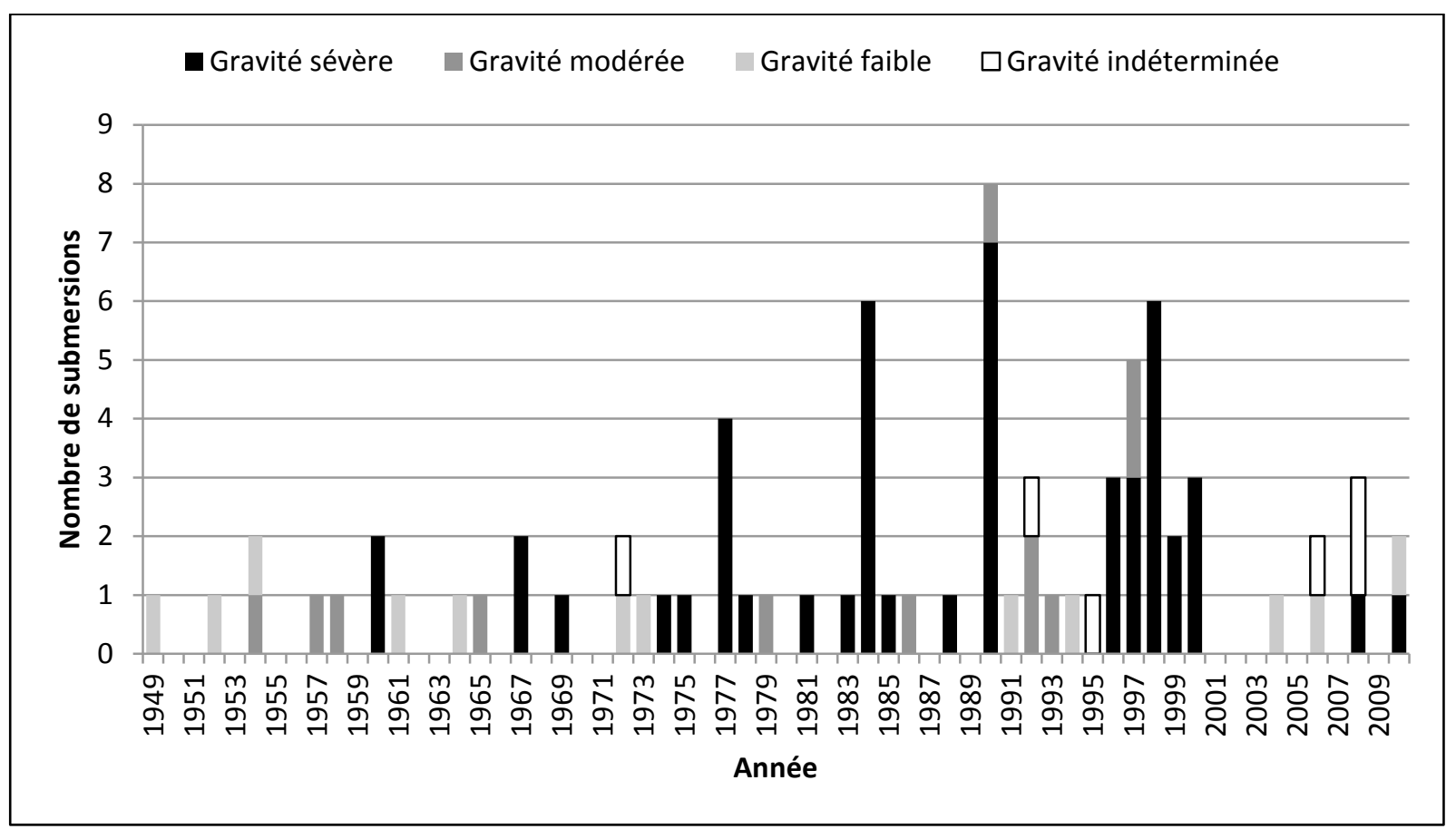

Figure 7 : Nombre de submersions recensées en Manche orientale de 1949 à 2010 selon leur degré de gravité. Number of coastal flooding events censed in the eastern English Channel from 1949 to 2010 according to severity degree.

D'un point de vue temporel, si l'évolution du nombre des inondations par la mer ne montre pas de tendance générale bien affirmée au cours des 62 dernières années, nous observons cependant une grande variabilité interannuelle du phénomène, marquée par une pulsation positive des submersions sévères à la fin des années 1980 déjà identifiée en Manche orientale (Costa, 1997). S'agissant de la fréquence des inondations par la mer, il convient d'être prudent car ces phénomènes sont la résultante d'une combinaison entre facteurs naturels (conditions météo-marines, stock sédimentaire), facteurs anthropiques (occupation humaine) et perception du phénomène (résonance journalistique). En raison d'une occupation humaine historiquement ancienne, la pulsation positive résulte probablement d'un contexte de crise sédimentaire favorisant un accroissement du nombre d'inondations par la mer. De plus, il est possible que ces phénomènes soient davantage perçus en raison d'une sensibilité accrue des sociétés aux problématiques environnementales.

D'un point de vue spatial, l'approche historique met également en évidence les sites particulièrement sensibles aux inondations par la mer (tous degrés de gravité confondus depuis 62 ans). Par ordre décroissant, pour les communes présentant un minimum de 10 submersions, nous retrouvons Mers-les-Bains, Dieppe, Criel-sur-Mer, Quiberville, Hautotsur-Mer, Étretat, Fécamp et Le Tréport (figure 8).

Afin de reconstituer l'ensemble des conditions météo-marines ayant provoqué le franchissement, chaque submersion a été étudiée en détail, de l'échelle locale (prise en compte des effets de site) jusqu'à l'échelle de l'Atlantique nord (cartes météorologiques synoptiques permettant d'appréhender la dynamique générale de l'atmosphère). 


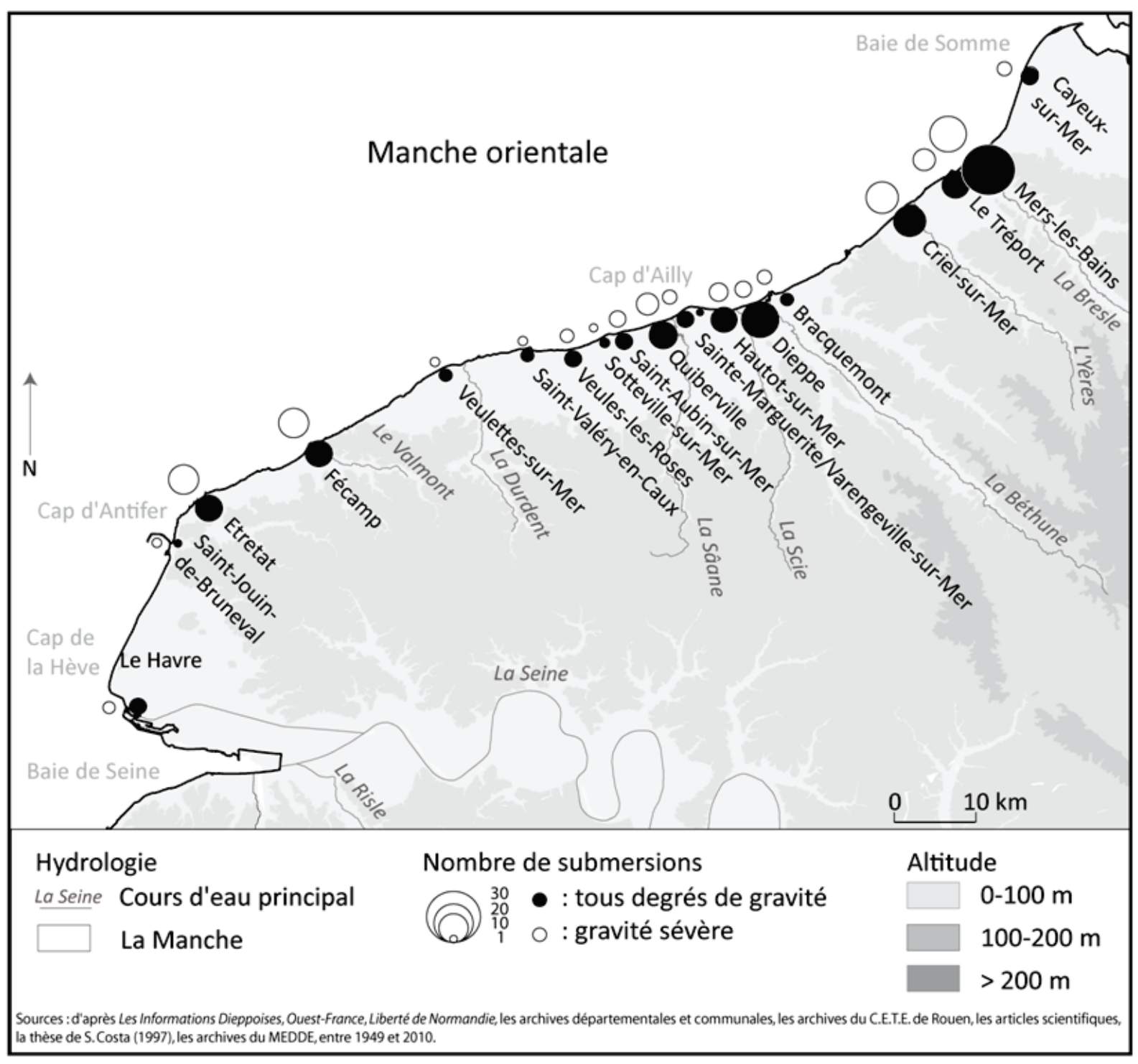

Figure 8 : Nombre de submersions marines recensées par commune entre Le Havre et Cayeux-sur-Mer (19492010). Number of coastal flooding events inventoried by commune between Le Havre and Cayeux-sur-Mer (1949-2010).

\section{Conditions météo-marines menant au franchissement en Manche orientale}

\subsection{Seuils anémométrique et marégraphique minimaux}

A l'échelle locale, cet inventaire nous autorise à définir les conditions minimales de vent et de hauteur d'eau à partir desquelles il y a risque d'inondation par la mer. Cette méthode est très souvent utilisée pour les systèmes d'alertes (Caspar, 1990 ; Costa, 1997). Pour garantir la fiabilité des résultats, l'analyse de la force et de la direction des vents mesurés s'est limitée à la période postérieure à 1981, conformément aux préconisations techniques de Météo-France (homogénéité des séries temporelles par harmonisation des instruments et des procédures de mesure).

La station Météo-France du cap de la Hève (Le Havre) et le sémaphore de Dieppe sont les deux seuls postes de mesures en contexte littoral sur le secteur d'étude. Préalablement à l'analyse numérique, ils ont été expertisés d'un point de vue métrologique et environnemental afin d'évaluer leur fiabilité et leur représentativité géographique. Concernant les données anémométriques, Stéphane Costa (1997) évoque un phénomène connu des prévisionnistes de 
la station du Havre, à savoir que la position de chacune des stations sous-estime des secteurs de vent. Le sémaphore dieppois minore les flux de sud, tandis que la station du cap de la Hève minimise ceux de nord. En raison de l'importance des conditions anémométriques préférentiellement d'afflux (ici d'ouest à nord-est), nous avons donc choisi comme référence le sémaphore de Dieppe. De plus, l'orientation sud-ouest / nord-est de son trait de côte est représentative de la grande majorité des côtes basses du secteur d'étude. Cette station se caractérise par des vents de sud fréquents mais peu violents alors que les flux de quadrant ouest et nord sont moins fréquents mais plus forts (figure 9).

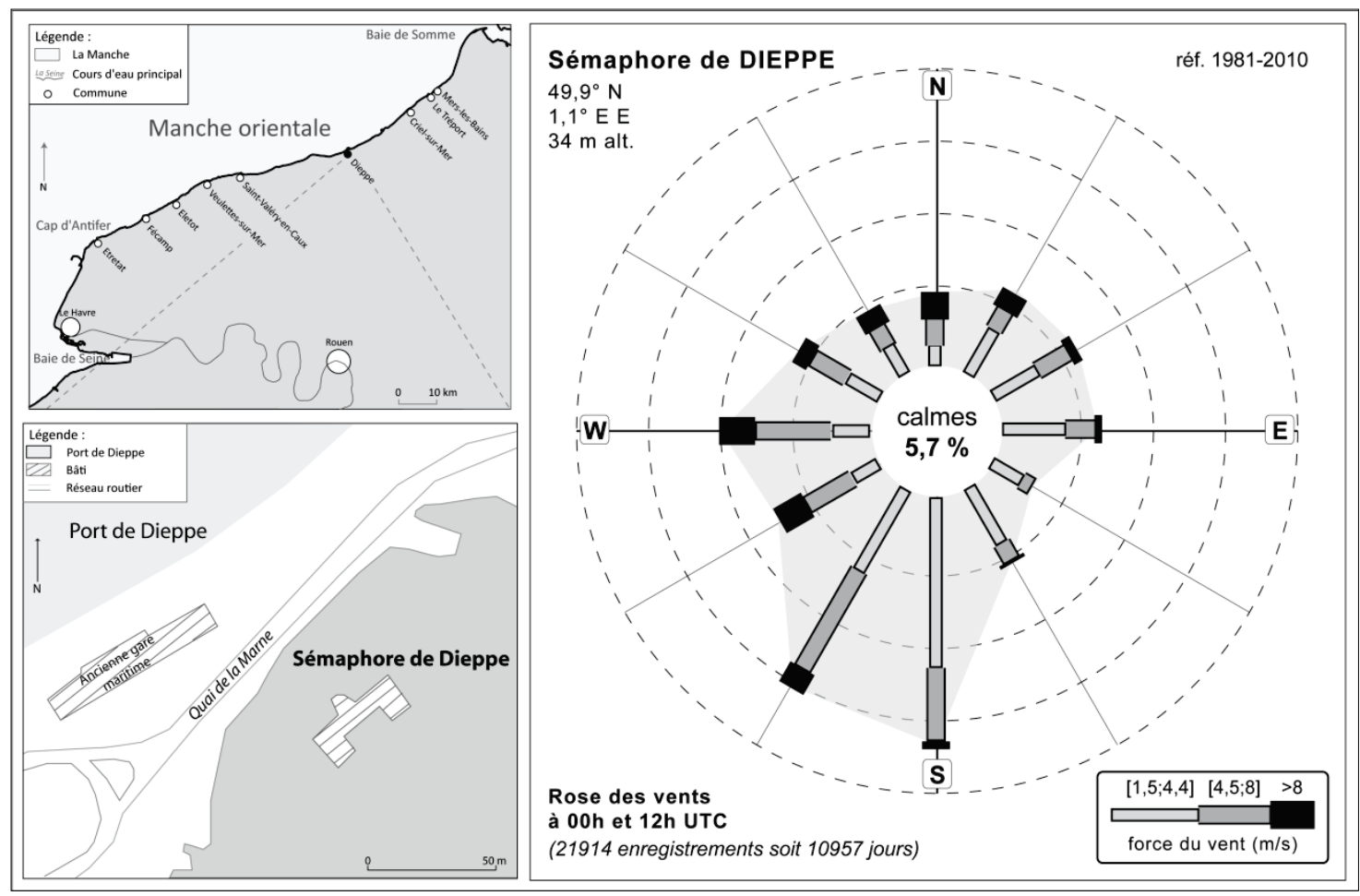

Figure 9: Localisation et rose des vents du sémaphore de Dieppe. Location and wind rose of the Dieppe meteorological measurement post, located at the national Navy semaphore.

Sur la période climatique aux données homogènes 1981-2010, soit une normale de 30 ans, nous comptabilisons 53 submersions parmi les 78 recensées dans l'inventaire initial 19492010. En plus des données aérologiques, l'existence de données marégraphiques disponibles à l'échelle locale (port de Dieppe) permet l'analyse complète des conditions météo-marines menant aux franchissements. Concrètement, il s'agit de définir les seuils aérologiques (force du vent donnée par Météo-France) et les seuils marégraphiques (hauteur d'eau prédite donnée par le $\mathrm{SHOM}^{4}$ ) au-delà desquels il y a risque de submersion.

Les niveaux de marée utilisés sont ceux prédits par le SHOM. La raison pour laquelle nous n'avons pas inclus la surcote ni même l'action du wave setup (accumulation d'eau à la côte induite par le déferlement) sont triples :

- il s'agit dans cet article de proposer des seuils minimums de franchissement. Il est donc plus aisé de déterminer, à partir des estimations du SHOM, les périodes dans l'année pendant lesquelles apparaissent des niveaux de marée pouvant potentiellement mener au franchissement ;

- l'indépendance des phénomènes que sont la marée et la surcote oblige à les dissocier dans l'estimation des hauteurs de franchissement;

\footnotetext{
${ }^{4}$ Service Hydrographique et Océanographique de la Marine.
} 
- le wave setup n'est pas pris en compte dans l'estimation des hauteurs car les seules données existantes sont celles issues des marégraphes implantés dans les ports. Or, dans ces zones d'abri, ce phénomène n'est pas ou peu enregistré. Leur calcul sur les plages de galets est par ailleurs extrêmement complexe et souffre d'une marge d'erreur importante.

Au regard de la figure 10, les seuils au moment du franchissement sont de l'ordre de $7 \mathrm{~m} / \mathrm{s}$ pour la force du vent et de $8,5 \mathrm{~m}$ pour la hauteur de la marée prédite à Dieppe.

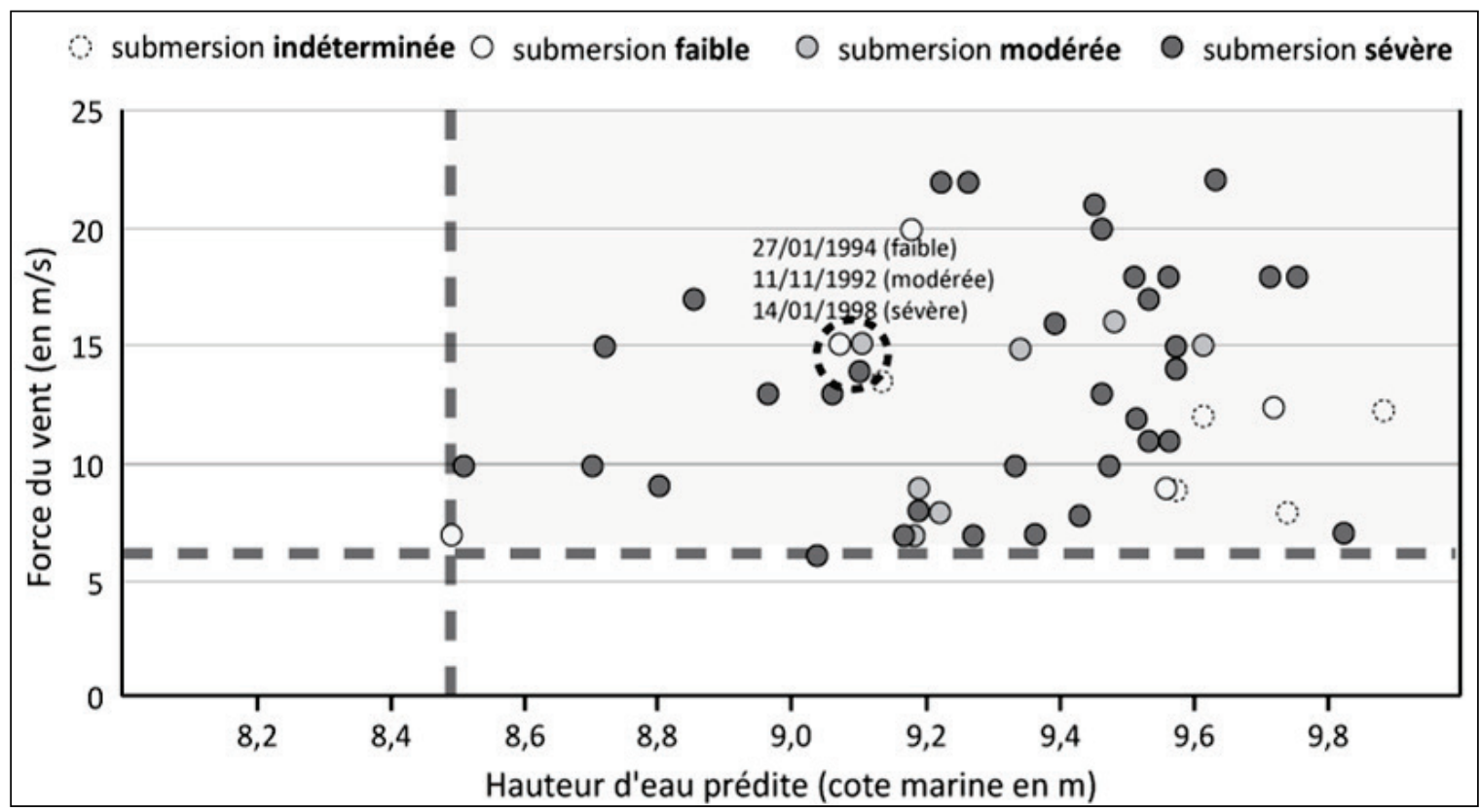

Figure 10: Submersion marine, hauteur de marée prédite et vitesse du vent (tri-horaire et horaire) à Dieppe (1981-2010). Coastal flooding event, forecast tide height and wind speed (three hourly and hourly) at Dieppe (1981-2010).

De plus, ce graphique montre que pour des seuils de marée et de vent équivalents, il est possible d'observer des submersions marines sévères, modérées et faibles. Cette situation suggère que d'autres facteurs que la hauteur de la marée et la force du vent sont susceptibles de contribuer à l'intensité des inondations par la mer.

\subsection{Le rôle du gradient de pression de nord-ouest : un indicateur plus global du flux atmosphérique}

Pour s'extraire des limites de fiabilité des données sémaphoriques et de leur manque de profondeur temporelle, il est possible de procéder à une bonne approximation de la force et de la direction du vent ${ }^{5}$ en analysant le gradient de pression à partir des cartes synoptiques. En raison de la configuration de la Manche et du dessin d'ensemble des côtes normano-picardes, les vents de dominante ouest sont les plus propices à la genèse de houles prononcées. Afin de qualifier et de quantifier ces flux à l'échelle de la Manche, nous avons étudié le gradient de pression de nord-ouest en surface. Pour aboutir à une évaluation globale et rapide sur un vaste espace maritime de ces situations barométriques, nous avons défini un transect de 750 kilomètres allant d'Ouessant (Bretagne) à Cromer (Norfolk), perpendiculaire aux flux de nord-ouest. L'analyse des cartes synoptiques des champs de pression de surface de l'ensemble des situations à submersion fait apparaître qu'il faut un gradient isobarique d'au moins 4

\footnotetext{
${ }^{5}$ Il s'agit du vent géostrophique, vent théorique qui résulterait de l'équilibre entre la force de Coriolis et la force du gradient de pression atmosphérique.
} 
intervalles (soit une différence de $20 \mathrm{hPa}$ entre les deux sites) pour qu'il y ait inondation par la mer (Costa, 1997).

Nous avons ainsi procédé à l'inventaire des situations synoptiques présentant un fort indice de gradient de pression de nord-ouest $(\geq 4)$ en nous appuyant sur les réanalyses NCEP (figure 11).

Figure 11: Gradient de pression de nord-ouest de $30 \mathrm{hPa}$ entre Ouessant et Cromer (Norfolk), soit 6 intervalles isobariques (indice 6) (situation du 26/02/1990, à 12 h UTC) (Costa, 1997). North-West pressure gradient of $30 \mathrm{hPa}$ between Ouessant and Cromer (Norfolk), so 6 isobaric intervals (index 6) (meteorological situation of 26/02/1990, at 12 h UTC).

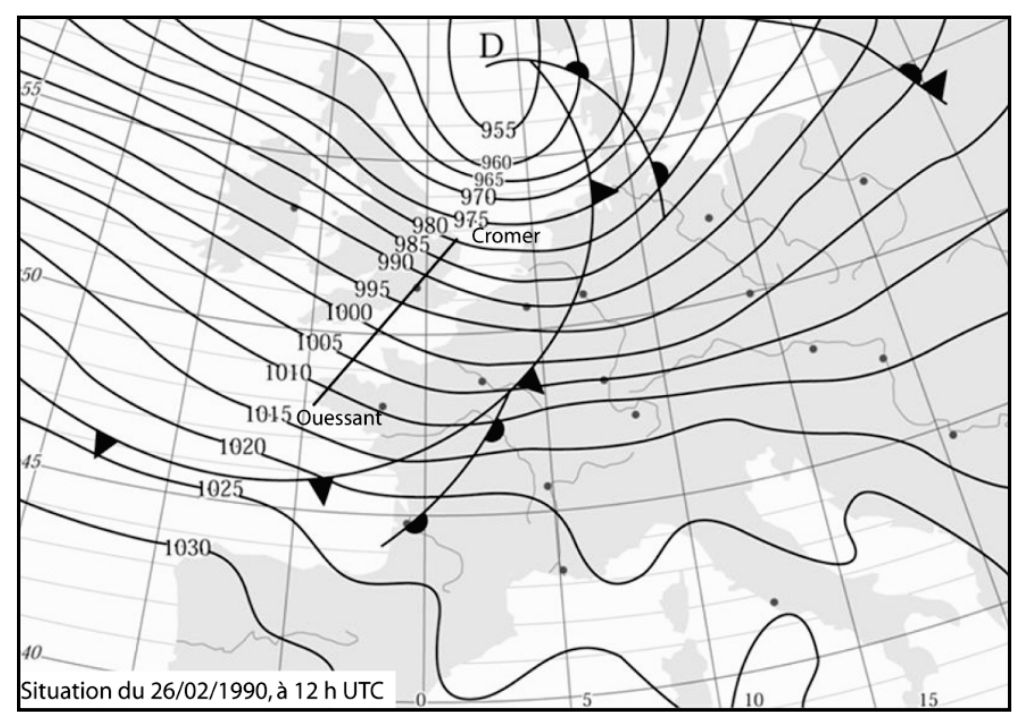

De 1949 à 2010, nous constatons que :

- parmi les submersions marines induites par les flux d'ouest à nord-ouest (69 cas), 28 sont induites par un gradient de pression de nord-ouest supérieur ou égal à 4, dont 20 sont sévères, 3 modérées, 3 de faible intensité et 2 indéterminées. Sur la période 19812010, 21 cas de submersions sont induits par un gradient de pression de nord-ouest supérieur ou égal à 4 ( 17 sévères, 3 modérées et 1 de faible intensité) ;

- le nombre de situations d'indice de nord-ouest supérieur ou égal à 4 connaît une forte variabilité interannuelle (figure 12). Là encore, aucune tendance ne semble se dégager, hormis quelques oscillations d'ordre décennal peu corrélées à l'Oscillation NordAtlantique (NAO - North Atlantic Oscillation) (figure 13);

- il n'existe pas de relation systématique entre le nombre de situations à fort gradient de pression de nord-ouest et l'occurrence des submersions marines. Par conséquent, d'autres critères explicatifs sont à prendre en compte.

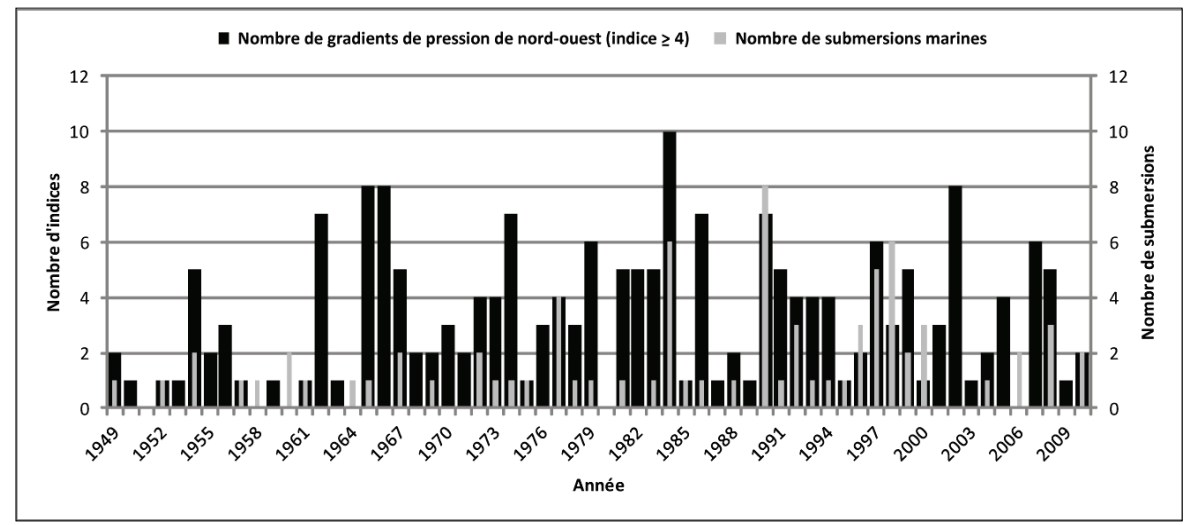

Figure 12 : Nombre de gradients de pression de nord-ouest (indice $\geq 4$ ) et de submersions marines en Manche orientale (1949-2010). Number of North-West pressure gradients (index $\geq 4$ ) and coastal flooding events in the eastern English Channel (1949-2010). 


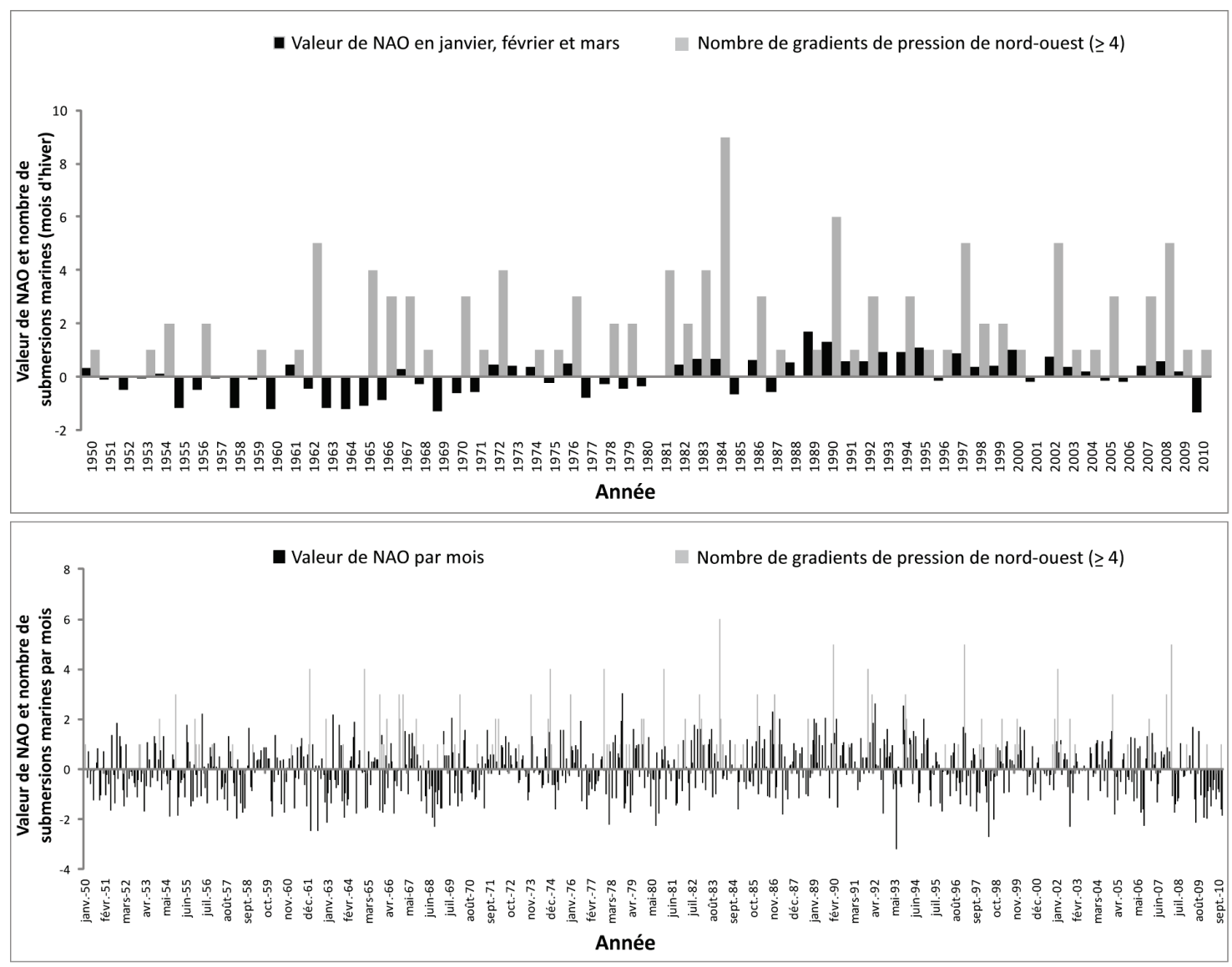

Figure 13 : NAO et nombre de gradients de pressions de nord-ouest $(\geq 4)$ (mois d'hiver et tous les mois de l'année). NAO and number of North-West pressure gradients (index $\geq 4$ ) (winter months and all months of the year).

Une des limites dans ce travail sur le gradient de pression nord-ouest est à évoquer. Le basculement des champs de pression ouest à nord-ouest peut se produire à un pas de temps parfois plus fin que le pas de temps des réanalyses NCEP ( $0 \mathrm{~h}$ et $12 \mathrm{~h}$ ). Il faudra, par la suite, inclure un gradient d'ouest à notre analyse, comme nous l'a montré empiriquement une analyse systématique de toutes les cartes synoptiques au sol et en altitude les jours précédant les franchissements. Pour pallier ce biais, une analyse par les données des réanalyses européennes ERA40 et ERA75 sera entreprise prochainement car ces données sont maintenant plus accessibles.

\subsection{Le passage du front froid au moment de la pleine mer : un facteur aggravant}

Aux latitudes moyennes, le flux d'ouest dominant est souvent animé par le passage de dépressions mobiles de front polaire, marquées par la succession de fronts chauds puis de fronts froids, traduisant le contact de masses d'air thermiquement contrastées (régime perturbé). Des études récentes sur le littoral normano-picard ont montré que $70 \%$ des inondations par la mer s'effectuent lors du passage d'un front froid actif (Costa et al., 2004 ; Pirazzoli et al., 2006 ; Caspar et al., 2007).

Dans de telles situations synoptiques, l'enchaînement des conditions atmosphériques est le suivant: à l'approche du front froid, la pression baisse considérablement et atteint son minimum devant le front, alors que dans le même temps le vent forcit progressivement. Par ailleurs, juste à l'arrière de ce front, la pression amorce une nette et rapide remontée tandis que le vent subit une rotation importante et tourne généralement à l'ouest, et même souvent au nord-ouest, restant très fort et avec de violentes rafales (figure 14). 


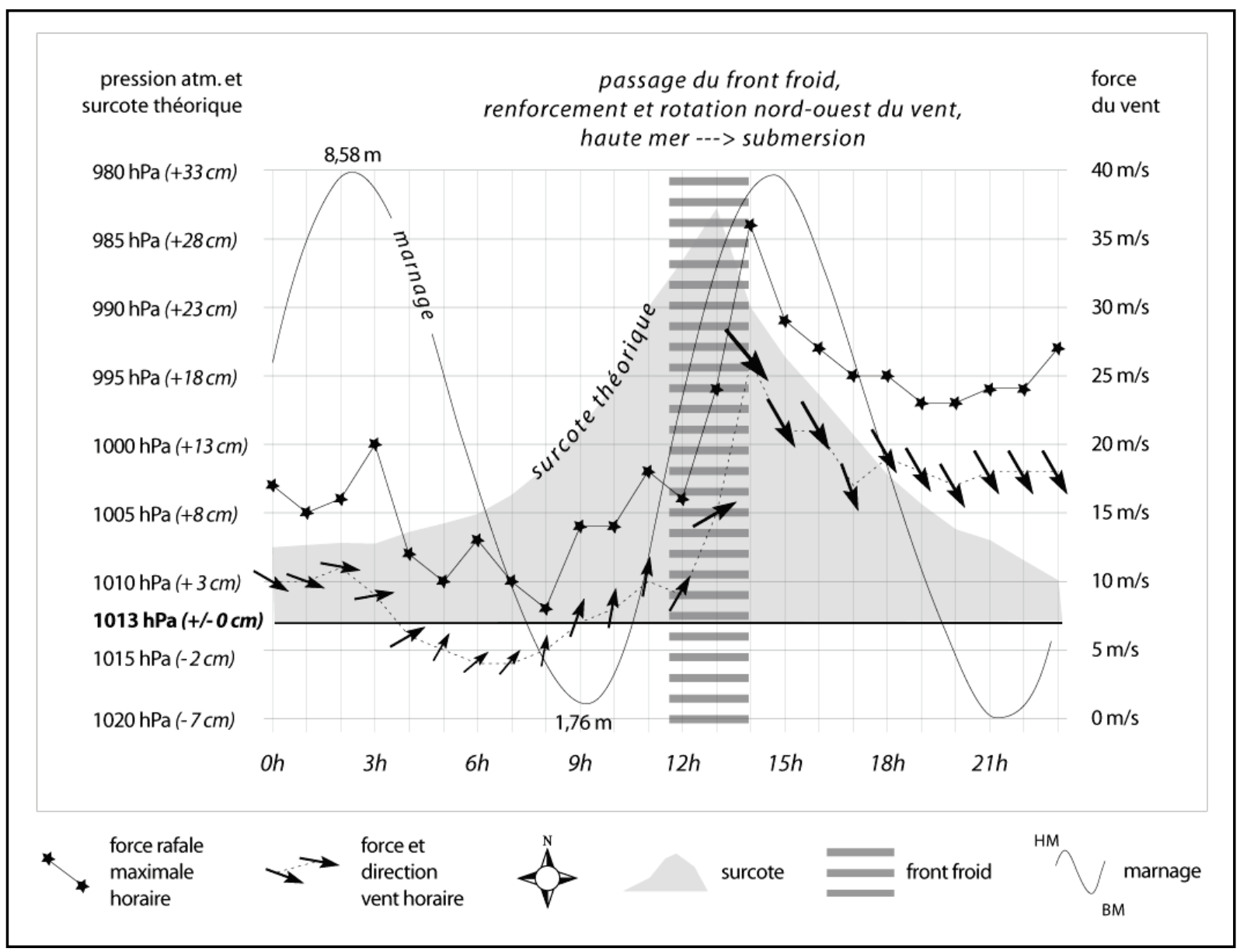

Figure 14 : Concomitance des éléments météo-marins lors d'une submersion marine : l'exemple du 17 décembre 2004 à Dieppe (données météorologiques horaires Météo-France et marégraphiques du SHOM). Combination of meteorological and marine elements for the coastal flooding event of 17/12/2004 in Dieppe (meteorological data from Meteo-France and marine data from SHOM).

Cette situation a deux conséquences : d'une part, une forte surcote se crée au droit du front froid en raison du minimum barométrique et, surtout, l'afflux d'eau à la côte augmente du fait $\mathrm{du}$ renforcement du vent et de sa rotation vers le nord-ouest. En outre, l'action du vent va augmenter la hauteur des vagues : d'après les abaques existants, un vent de $20 \mathrm{~m} / \mathrm{s}$ agissant sur un fetch de $30 \mathrm{~km}$ lèvera une houle de près de $2 \mathrm{~m}$ en moins de 2 heures. Le front froid ayant une certaine "épaisseur » horizontale, son action peut se faire durant 2 à 3 heures (Costa, 2005).

Enfin, à la faveur du changement brutal de direction du vent, le passage du front froid peut former une houle croisée - chaotique et très cambrée - particulièrement efficace pour le démaigrissement des plages et la projection d'eau au-delà des ouvrages (Costa, 2005 ; Caspar et al., 2010).

L'étude du front froid, à l'échelle de la Manche, a été effectuée grâce à la consultation :

- des BQE, bulletins quotidiens d'études (06 h, 18 h UTC) de 1949 à 1952 ;

- des BME, bulletins météorologiques européens ( 00 h et 12 h UTC) de 1952 à 2004 ;

- des bulletins Météo Hebdo (12 h UTC) et des bulletins d'UKMO (00 h UTC) sur le site internet de la Wetterzentrale de 2005 à 2008 ;

- des bulletins d'UKMO (00 h UTC) sur le site internet de la Wetterzentrale et des isofronts de Météo-France ( 06 h, 12h et 18 h UTC) de 2009 à 2010. 
La position du front froid peut avoir des conséquences variées le long du littoral en Manche. En effet, sa progression peut être en phase ou non avec celle de l'onde de marée. Les vitesses de déplacement du front froid et de l'onde de marée ne sont pas similaires. Cependant, la conjonction en un point donné est favorable à de forts franchissements.

Parmi les 53 submersions analysées sur la période de 1981 à 2010, 37 ont lieu lors du passage du front froid ( 4 de faible intensité, 6 modérées, 22 sévères et 5 d'intensité indéterminée). Six autres cas sont expliqués par le fort gradient de pression de nord-ouest. Notons qu'un fort gradient de pression de nord-ouest peut se combiner au passage du front froid, aboutissant ainsi à des submersions généralement sévères (15 cas dont 1 faible, 2 modérés, 11 sévères et 1 indéterminé). La combinaison des deux éléments est donc un facteur aggravant en termes d'impact à la côte. Le gradient de pression de nord-ouest et le passage du front froid correspondent à la phase terminale d'un processus complexe menant au franchissement. La chronologie est donc la suivante : (1) la mer se forme par flux d'ouest et s'engouffre en Manche puis, (2) si le flux vire au nord-ouest (généralement après le passage du front froid), il y a (3) risque de franchissement par « rabattement » car les côtes forment alors un obstacle perpendiculaire au flux sur lequel vient buter l'énergie marine.

Lorsque nous combinons tous les précédents éléments, 10 submersions ne s'expliquent ni par un fort gradient de pression de nord-ouest, ni par le passage d'un front froid. Un autre facteur explicatif doit donc être pris en considération.

\section{Le rôle des conditions météo-marines préalables à la submersion}

L'analyse des seules conditions météo-marines au moment du franchissement n'étant pas suffisante pour expliquer la totalité des événements recensés, il est nécessaire d'élargir notre analyse aux heures et aux jours précédant la submersion.

\subsection{L'adaptation morpho-sédimentaire des plages de galets haut-normandes et picardes}

Il est souvent fait référence dans la littérature qu'au-delà des conditions météo-marines menant au franchissement, il faut prendre en considération les conditions morphosédimentaires des plages pour expliquer les inondations par la mer (Orford, 1977 ; Paskoff, 1985 ; Carter, 1988 ; Carter et Orford, 1993 ; Costa, 1997 ; Sabatier et al., 2009 ; Cariolet et al., 2010). En effet, un budget sédimentaire déficitaire et un profil de plage abaissé sont des facteurs aggravants et fortement favorables aux franchissements.

La dimension moyenne des galets constituant les plages haut-normandes est de l'ordre de 40 à $75 \mathrm{~mm}$ et ne dépasse que rarement $140 \mathrm{~mm}$. Le suivi granulométrique effectué sur quelques unes de ces plages montre que la répartition des sédiments dans le profil peut évoluer entre deux marées. En effet, l'agencement sédimentaire de surface des plages de galets est très variable dans le temps et l'espace en réponse à l'évolution de l'agitation. Cette situation résulte de l'existence de sédiments de taille et de forme très variées (plages de type composite), en surface et en profondeur, réagissant de manière différenciée aux conditions d'agitation, elles-mêmes changeantes selon la hauteur d'eau et la morphologie de la plage (Mason et al., 1997 ; Carter, 1988 ; Costa, 1997 ; Morel, 1997 ; Mason et Coates, 2001 ; Orford et al., 2002; Pontee et al., 2004 ; Costa et al., 2008 ; Stéphan, 2008). De plus, contrairement au fonctionnement des barrières de galets, les plages haut-normandes ont un fonctionnement très spécifique en raison de la présence d'un ouvrage longitudinal de haut estran (perré) sur lequel les vagues de tempête sont fortement réfléchies (Paskoff, 1985; Carter, 1988 ; Costa, 1997 ; Morel, 1997 ; Orford et al., 2001). La réflexion de la houle sur les perrés produit des tris granulométriques, comme par exemple l'apparition de la fraction sableuse en surface après certaines tempêtes (Costa, 1997 ; Costa, 2005 ; figure 15).

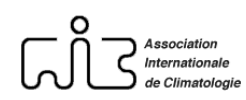




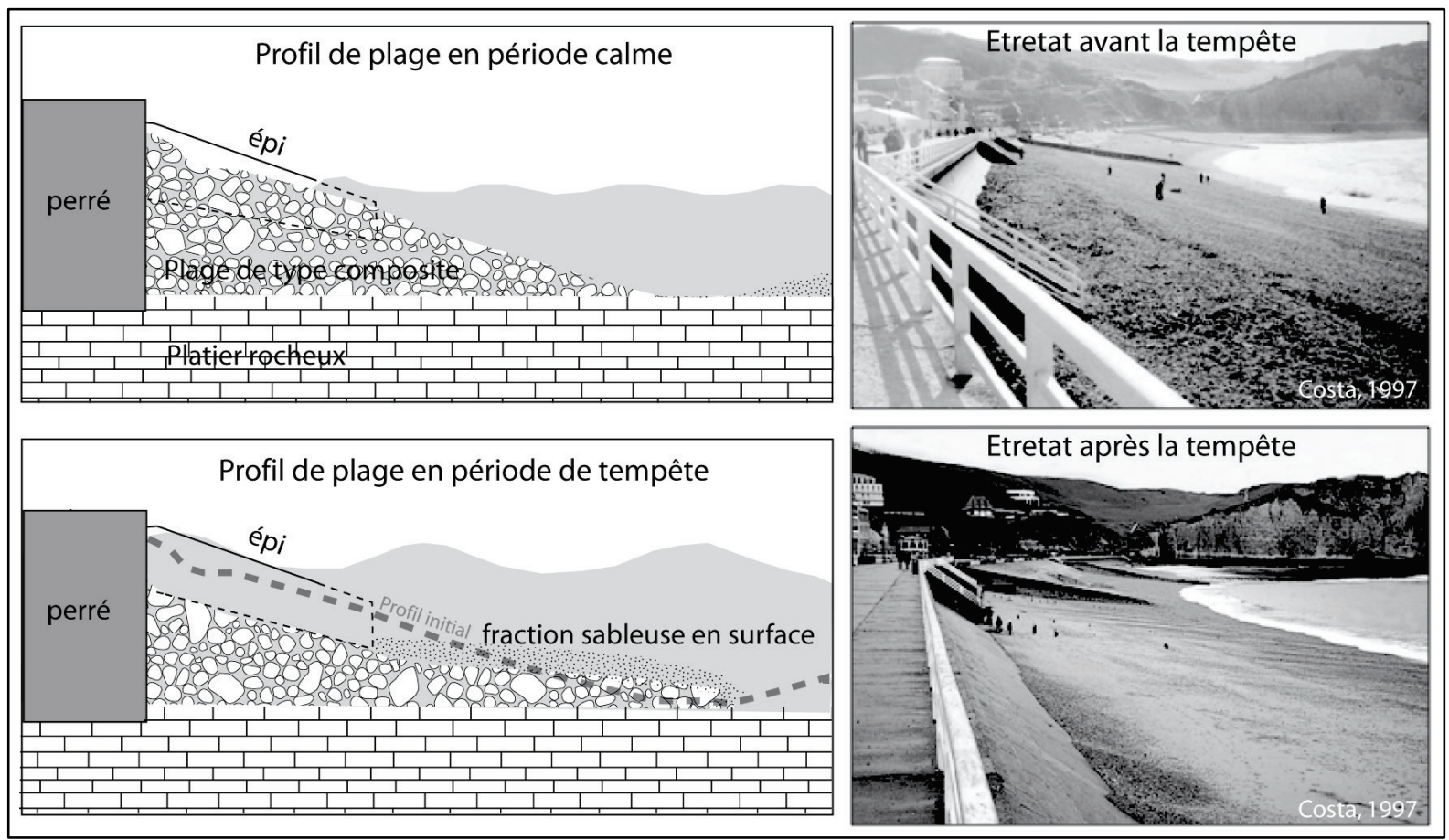

Figure 15 : Evolution du profil de plage d'Etretat en fonction des conditions météo-marines : avant la tempête et après la tempête. Evolution of the beach profile at Etretat in function of meteorological and marine conditions: before and after storm.

Pour ces diverses raisons, nous nous sommes intéressés à l'état des stocks de sédiments sur les plages peu avant l'inondation par la mer. L'absence de suivi topographique régulier nous a amenés à observer les conditions anémométriques et donc d'agitation pouvant générer des abaissements de profil de plage. En effet, d'anciens levés de profils de plage de galets (Costa, 1997 ; Costa et al., 2008) montrent l'existence d'importantes variations altimétriques lors de forts coups de vent, pouvant atteindre 3 mètres à leur sommet lors d'un seul cycle tidal (Costa, 1997 ; Costa, 2005 ; Caspar et al., 2010).

Le seuil de vent considéré comme susceptible de générer des abaissements du profil de plage dépasse $8 \mathrm{~m} / \mathrm{s}$ (vent fort selon Météo-France). Au-delà de la force du vent, nous avons intégré la composante direction du vent à cette analyse (données anémométriques de 1981 à 2010). En effet, seules les directions de vent en composante d'afflux, susceptibles de générer des vagues à la côte, sont analysées. Le secteur de vent jugé efficace est [230-060 ]. Le pas d'analyse s'étend sur les 28 jours précédant la submersion pour adopter une démarche exploratoire qui permet de prendre en compte l'inertie du système côtier au cours d'un cycle de marée complet.

Deux situations sont observées (figure 16):

1) des vents forts et fréquents tout au long des 28 jours analysés (cumul minimum de $80 \mathrm{~h}$ de vents forts) : 5 cas ;

2) des vents forts mais concentrés sur les dernières $48 \mathrm{~h}$ avant le franchissement (minimum de $12 \mathrm{~h}): 5$ cas.

Ce temps de préparation morphologique de la plage est particulièrement intéressant à étudier pour les submersions sévères à la limite des seuils minimaux de force de vent et de hauteur de marée prévue au-delà desquels il y a risque de submersion. La figure 16 met en évidence les différents temps de préparation pour les deux submersions sévères en limite de seuil $(25 / 10 / 1998$ et $25 / 11 / 1984)$ : 


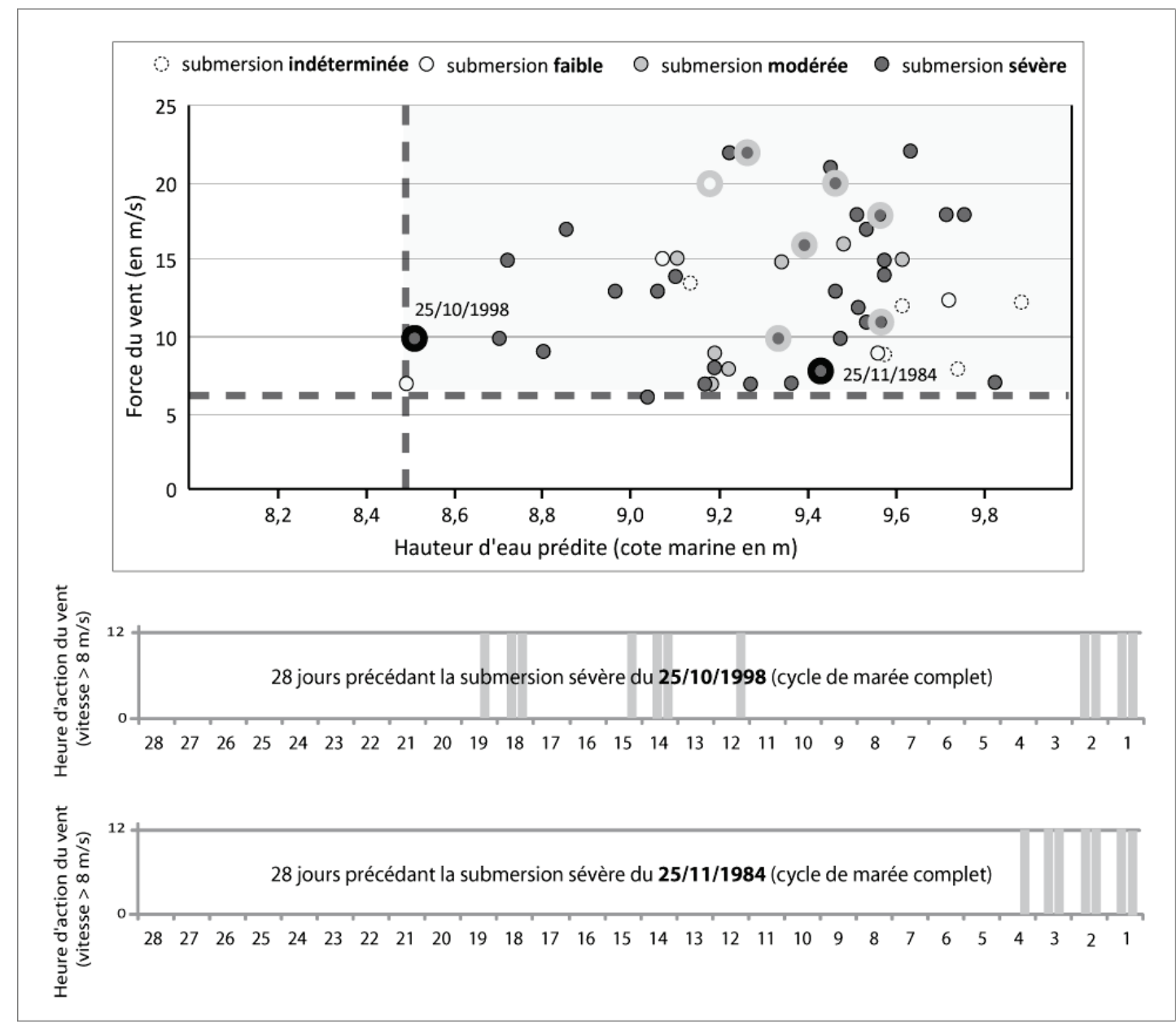

Figure 16 : Submersions inexpliquées (cercle gris) et celles sévères du 25/10/1998 et du 25/11/1984 (cercle noir) et leurs temps de préparation. Unexplained coastal flooding events (grey circle) and severe unexplained coastal flooding events of 25/10/1998 and 24/11/1984 (black circle) and their preparation time.

- la submersion sévère du 25/10/1998 nécessite une longue durée d'action de vent fort « efficace » $\left[230-060^{\circ}\right]$. En effet, la plage doit être fortement abaissée pour que malgré une hauteur de marée et une force du vent relativement faibles, la submersion soit sévère.

- la submersion sévère du 25/11/1984 nécessite une plus courte durée d'action de vent d'afflux fort. La plage a été au préalable abaissée par des vents forts et continus sur $88 \mathrm{~h}$ (provoquant deux submersions, le 23 et 24/11/1984). Ces 88 h sont efficaces en termes d'abaissement du profil de plage, favorisant un franchissement sévère.

Ces résultats corroborent ceux observés en milieu sableux sur la côte d'Opale (région Nord-Pas-de-Calais) et en Bretagne (Suanez et Cariolet, 2010 ; Maspataud, 2011).

Il est important de préciser qu'à l'inverse les conditions morpho-sédimentaires ont parfois empêché la survenue d'une submersion marine ou limité leur intensité (en cas d'exhaussement du profil de plage).

\subsection{Trajectoires des centres dépressionnaires menant à la submersion : une base de prévision}

Afin d'aboutir à une approche prospective, nous avons analysé l'évolution des 78 inondations par la mer en étudiant, à l'échelle de l'Atlantique nord et sur les 2 jours précédant l'inondation, les positions successives - et donc les trajectoires - des centres dépressionnaires 
à l'origine des submersions. L'objectif est de mettre en évidence d'éventuels types de trajectoires favorables aux franchissements. Ce travail complète une approche initialement fondée sur les types de circulation GWL (Grosswetterlagen) qui avait l'inconvénient de proposer une fenêtre temporelle et spatiale trop large pour prendre en compte les subtilités de la dynamique atmosphérique nécessaires à notre problématique.

Pour des raisons d'homogénéité des données sur l'ensemble de la période 1949-2010, notre travail s'est appuyé sur les réanalyses de la NCEP (National Centers for Environmental Prediction) du champ barométrique au sol et en altitude et des vents résultants, à $00 \mathrm{~h}$ et $12 \mathrm{~h}$ UTC. Ces réanalyses, dont la résolution spatiale est de $2,5^{\circ}$ x $2,5^{\circ}$, sont accessibles sur le site internet originel de la NOAA (National Oceanic and Atmospheric Administration) et reprises sur des sites français tels que Météociel ou Infoclimat.

Sur les 78 submersions recensées, deux grands types de trajectoires sont mis en évidence (figure 17) : les trajectoires de quadrant ouest (75 cas) et celles de quadrant nord (3 cas). Au sein du «quadrant» ouest, l'écrasante majorité des submersions (69 cas) possède une trajectoire d'ouest contre 6 cas de sud-ouest. Cette prépondérance est néanmoins caractérisée par une très grande variabilité spatiale des positions successives du centre de la dépression.

Malgré cette variabilité, deux grands types de circulation d'ouest sont mis en évidence, pouvant ainsi améliorer la prévision/prévention de l'aléa :

1) une circulation rapide d'ouest, à hauteur de la Manche, d'une dépression qui s'accompagne de vents de nord-ouest en position de traîne (15 cas) ;

2) une pénétration en Mer du Nord d'une dépression atlantique de plus haute latitude, se dirigeant vers la Scandinavie (46 cas). Le gradient de pression se renforce alors sur la Manche. Des forts vents de nord-ouest se lèvent et persistent tant que la dépression ne s'éloigne pas ou ne se comble pas (Costa, 1997).
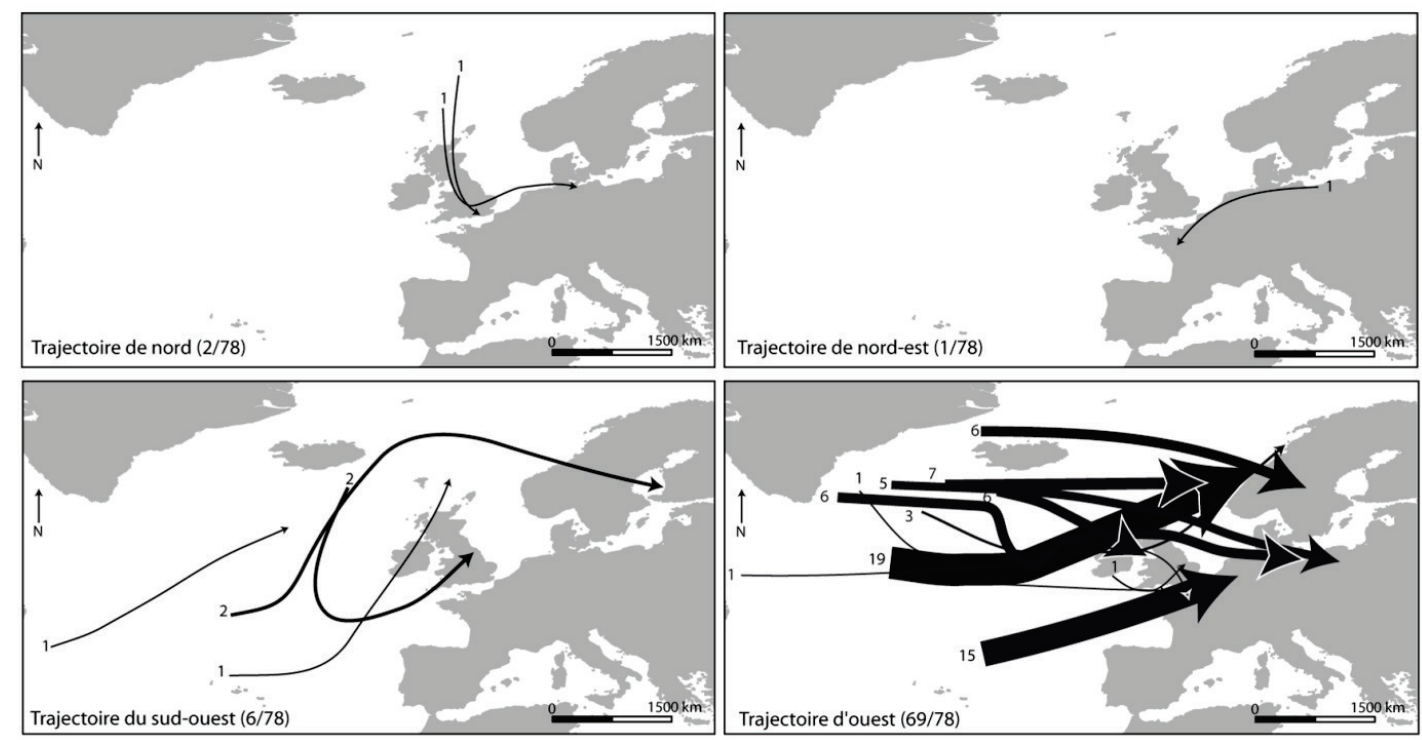

Figure 17: Trajectoires des centres dépressionnaires ayant provoqué des submersions (toutes intensités confondues) (l'épaisseur de la flèche varie en fonction du nombre de submersions marines). Trajectories of low air pressure centres ending in coastal flooding events (all degrees of severity) (thickness of the arrow evolves in function of the number of coastal flooding events).

Ces résultats renvoient au modèle explicatif des perturbations atlantiques (Joly, 1995) qui met en évidence le déplacement des dépressions d'ouest en est le long d'un rail (configuration 
zonale). L'étude des trajectoires des dépressions d'origine atlantique met en évidence deux configurations. Dans la première, le rail qui part toujours de la région de Terre-Neuve, se coupe en deux au niveau du $30^{\text {ème }}$ méridien ouest pour se diriger vers l'Islande ou vers l'Espagne (figure 18A).

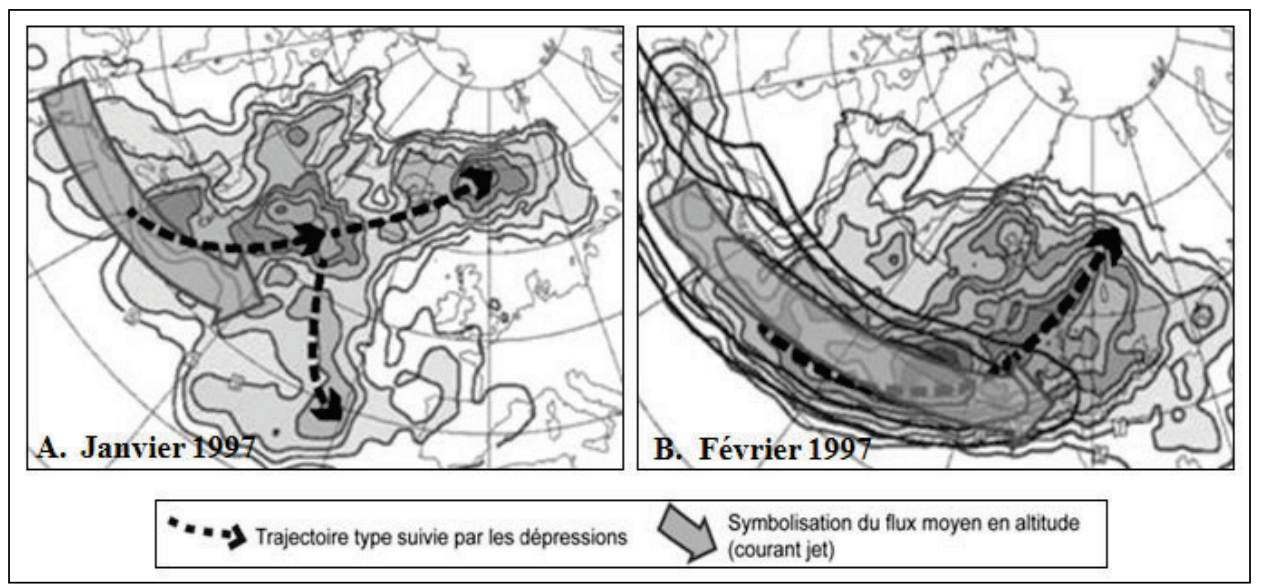

Figure 18 : A) Le rail des dépressions en janvier 1997, avec une configuration de blocage : les tempêtes restent sur l'Atlantique. Le courant-jet, courant d'altitude, est limité à la partie ouest de l'océan Atlantique (d'après C. Baehr et al., 1999 ; source : http://www.cnrm.meteo.fr/dbfastex/recyf_temp/tempet003.html\#railbloc). Rail of low air pressure in January 1997. The meteorological configuration is "blocked": storms stay in Atlantic zone. The jet-stream is confined to the West part of the Atlantic Ocean (from Baehr et al., 1999; source: http://www.cnrm.meteo.fr/dbfastex/recyf_temp/tempet003.html\#railbloc). B) Le rail des dépressions en février 1997. Dans cette configuration dite «zonale », le rail des dépressions s'étend le long du $50{ }^{\text {ème }}$ parallèle. Les dépressions ne sont pas bloquées. On remarque que le courant-jet s'étend plus à l'est et que les dépressions ont tendance à couper le courant-jet du côté de son extrémité est (d'après C. Baehr et al., 1999; source : http://www.cnrm.meteo.fr/dbfastex/recyf_temp/tempet004.html\#railzonal). Rail of low air pressure in February 1997. In this "zonal" configuration, the rail of low air pressure extends along the $50^{\text {th }}$ parallel North. Low air pressure is not blocked. We notice that jet-stream extends to East and low air pressure can cut the eastern extremity of the jet-stream (from Baehr et al., 1999; source: http://www.cnrm.meteo.fr/dbfastex/recyf_temp/tempet003.html\#railbloc).

Dans la seconde configuration, la plus fréquente, le rail s'étend le long du $50^{\text {ème }}$ parallèle jusqu'aux îles Britanniques (Bessemoulin, 2002 ; Beltrando, 2004). Leurs recherches ont également permis d'identifier le rôle des courants-jets. Ces courants d'altitude générés par la différence de température entre les pôles et l'équateur, circulent d'ouest en est aux latitudes $50-55^{\circ} \mathrm{N}$ (régions tempérées) dans la haute troposphère $(8-10 \mathrm{~km}$ d'altitude) et favorisent la formation et le creusement des dépressions. Ce phénomène s'explique en partie par le mécanisme d'interaction barocline, défini comme étant l'amplification de deux anomalies (une anomalie froide située en altitude et une anomalie de surface, à savoir une dépression) qui interfèrent avec le courant-jet (Ayrault et Joly, 2000). La région la plus favorable à l'amplification des tempêtes semblerait se situer à l'extrémité est du jet (figure 18B). En configuration zonale, les dépressions coupent ainsi souvent le courant-jet au niveau du $15^{\text {ème }}$ méridien ouest, et s'en trouvent alors amplifiées par le mécanisme d'interaction barocline (Ayrault et Joly, 2000 ; Bessemoulin, 2002 ; Cariolet et al., 2010). Une analyse de la position et de l'intensité des courants jets et des submersions par la mer est actuellement en cours.

\section{Conclusion}

Grâce à un travail d'inventaire réalisé à partir de sources diverses entre 1949 et 2010, l'étude des submersions marines en Manche orientale s'est appuyée sur des événements d'intensité variée. Cette prise en compte d'événements extrêmes (submersion sévère) et nonextrêmes (submersion faible et modérée) a permis de mettre en évidence deux éléments.

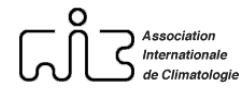


D'une part, l'absence de tendance évolutive significative de la fréquence de ces situations au cours des soixante dernières années, même si quelques oscillations pluriannuelles s'individualisent nettement, faisant plus appel à la variabilité climatique, inhérente à nos latitudes, qu'à une variation sur le long terme. A cette explication «naturelle » nous devons ajouter la dimension anthropique du problème, liée à l'augmentation de la vulnérabilité des côtes en raison du développement de nouvelles constructions et au phénomène de résonance journalistique.

D'autre part, la genèse de ces phénomènes météo-marins est complexe. Leur compréhension bénéficie d'une démarche inductive et naturaliste (sans a priori sur les facteurs explicatifs) et d'une approche par emboîtement d'échelles spatiales (de la position des centres d'action, au suivi de la position du front froid ou de la surcote par rapport à l'onde de marée) et d'échelles temporelles (de la submersion, jusqu'aux 28 jours la précédant) mais également systémique (interaction entre divers processus météo-marins et entre forme de plage et processus hydrodynamiques). Ainsi, à l'échelle de la Manche, l'influence du gradient de pression et du passage du front froid ont pu être mis en évidence. Cependant, certaines submersions ne peuvent être expliquées uniquement par ces facteurs-là. Pour les comprendre, il est nécessaire d'observer les conditions d'agitation sur les 28 jours précédant la submersion, afin d'intégrer le budget sédimentaire des plages et leur profil. L'évolution morphologique de ces plages peut être extrêmement rapide. Cette étude a permis de mettre en évidence qu'une forte agitation marine au cours des 48 heures précédant l'inondation par la mer peut suffire pour générer un abaissement du profil de plage, synonyme d'un rapprochement à la côte du point de déferlement des vagues de tempête.

Quant à la prévision, l'étude a permis d'identifier deux trajectoires principales des centres d'action majoritairement impliquées dans les submersions génératrices de dommages. Ces trajectoires sont prévisibles quelques jours à l'avance et intégrées dans les modèles de prévision de Météo-France. Afin d'affiner la compréhension des phénomènes générateurs de submersions marines, il conviendra d'utiliser des documents à une échelle spatiale et à des pas de temps plus fins. De plus, il sera nécessaire d'intégrer les prédictions du changement climatique. En effet, pour le climat des tempêtes aux latitudes moyennes, trois changements importants sont évoqués (Keim et al., 2004 ; Lozano et al., 2004 ; Wang et al., 2011) : un déplacement vers les pôles de trajectoires des tempêtes des moyennes latitudes (Meehl et al., 2007); un nombre croissant de tempêtes pour certaines régions (la Grande Bretagne par exemple); une réduction mineure du nombre total de dépressions (par exemple Lambert et Fyfe, 2006 ; Leckebusch et al., 2006 ; Loeptien et al., 2008 ; Ulbrich et al., 2009). Pour les régions telles que les îles Britanniques/mer du Nord ou l'Europe de l'ouest, une tempête de période de retour de 20 ans pourrait devenir un événement avec une période de retour de 10 ans autour de 2030-2040, et un événement de période de retour de 5-6 ans en 2100 (DellaMarta et Pinto, 2009). De tels changements dans le climat des tempêtes pourraient donc avoir des impacts considérables sur les sociétés et la dynamique des milieux littoraux.

Ces possibles changements seront prochainement abordés grâce aux données de l'ANRSCAMPEI (2011) qui, à partir de trois modèles de climats à maille fine (ALADIN, LMDZ et MAR) et de trois scénarios de concentration de gaz à effets de serre préconisés par le GIEC (A1B, A2 et B1), nous fournissent notamment des données de vent (moyen et rafales maximales) sur un futur proche (2030) et un futur lointain (2080).

Remerciements : Les auteurs remercient les relecteurs anonymes pour leur contribution à l'amélioration du manuscrit, Météo-France et le SHOM pour l'accès à leur banque de données, et Blandine Parey pour ses relectures. 


\section{Bibliographie}

AGASSE E., 2005 : Les crues de rivières en Basse-Normandie. Expression d'un système hydrogéographique complexe en milieu tempéré océanique. Thèse de Géographie, Université de Caen Basse-Normandie, 551 pages.

ALLEN H., DELANNOY B., 1990 : Mesure de houle en différents sites du littoral français. Rapport EDF-LNH HE-45/88.03.532, 288 pages.

ANSELME B., GOELDNER-GIANELLA L., DURAND P., 2008 : Le risque de submersion dans le système lagunaire de La Palme (Languedoc, France) : nature de l'aléa et perception du risque. Colloque international pluridisciplinaire "Les littoraux : subir, dire et agir.

AYRAULT F., JOLY A., 2000 : Une nouvelle typologie des dépressions atmosphériques : classification des phases de maturation. Comptes Rendus de l'Académie des Sciences, Sciences de la terre et des planètes, 330, 167-172.

BAEHR C., POUPONNEAU B., AYRAULT F., JOLY A., 1999: Dynamical characterization of the FASTEX cyclogenesis cases. Quarterly Journal of the Royal Meteorological Society, 125, 3469-3494.

BELTRANDO G., 2004 : Les climats : processus, variabilité et risques. Paris, Ed. Armand Colin, 261 pages.

BESSEMOULIN P., 2002 : Les tempêtes en France. Annales des Mines, 9-14.

BRUZZI C., 1998 : Les tempêtes et l'évolution morphosédimentaire des plages orientales du delta du Rhône. Thèse de Géographie, Université de Provence, 325 pages.

Bulletin Météorologique Européen (BME) (0 h, 12 h UTC).

CANTAT O., SAVOURET E., BRUNET L., 2009 : Les anémomorphoses végétales : quelle signification géoclimatique réelle? Climatologie, 6, 9-31.

CARIOLET J.-M., 2011. Inondation des côtes basses et risques associés en Bretagne. Vers une redéfinition des processus hydrodynamiques liés aux conditions météo-océaniques et des paramètres morpho-sédimentaires. Thèse de Géographie, Université de Bretagne Occidentale, 348 pages.

CARIOLET J.-M., SUANEZ S., 2009 : Approche méthodologique pour une cartographie du risque de submersion des côtes basses. La Houille Blanche, 2, 52-58.

CARIOLET J.-M., COSTA S., CASPAR R., ARDHUIN F., MAGNE R., GOASGUEN G., 2010 : Aspects météo-marins de la tempête du 10 mars 2008 en Atlantique et en Manche. Norois, 215, 11-31.

CARTER R. W. G., 1988 : Coastal environments: An introduction to the physical, ecological and cultural systems of coastlines. London, Academic Press, 617 pages.

CARTER R. W. G., ORFORD J. D., 1993 : The morphodynamics of coarse clastic beaches and barriers: A short- and long-term perspective. Journal of Coastal Research, 15, 158-179.

CASPAR R., 1988 : Marée de tempête sur le littoral de Haute-Normandie. 22 janvier 1988. Met. Mar, 141, 12-18.

CASPAR R., 1990 : Marées de tempête dans le nord-ouest de la France. Neptunia, Amis des musées de la Marine, 178, 8-15.

CASPAR R., POULLAIN H., 1996 : Coups de vent de nord-est sur les côtes normandes. Etudes de la situation des 19 et 20 février 1996. Met. Mar., 172, 33-38. 
CASPAR R., COSTA S., JACOB E., 2007 : Fronts froids et submersions de tempête dans le nord-ouest de la France : le cas des inondations par la mer entre l'estuaire de la Seine et de la Somme. La Météorologie, 57, 37-47.

CASPAR R., COSTA S., LEBRETON P., LETORTU P., 2010 : Les submersions de tempête de la nuit du 10 au 11 mars 2008 sur la côte d'Albâtre (Haute-Normandie, France) : détermination météo-marine. Norois, 215, 115-132.

COSTA S., 1997 : Dynamique littorale et risques naturels : l'impact des aménagements, des variations du niveau marin et des modifications climatiques entre la Baie de Seine et la Baie de Somme. Thèse de Géographie, Université de Paris I, 376 pages.

COSTA S., 2005 : Falaises à recul rapide et plages de galets : de la quantification des dynamiques d'un système complexe à la caractérisation des risques induits. Habilitation à Diriger les Recherches, Université de Bretagne Occidentale, 310 pages.

COSTA S., CANTAT O., PIRAZZOLI P. A., LEMAITRE M., DELAHAYE D., 2004 : Vents forts et submersions de tempête en Manche orientale : analyse météo-marine sur la période historique récente. Actes du Colloque de l'Association Internationale de Climatologie " Climat, mémoire du temps. Les relations climat-espace-société », 277-280.

COSTA S., HENAFF A., LAGEAT Y., PLESSIS E., 2002 : Le recul des falaises crayeuses du Pays de Caux : détermination des processus d'érosion et quantification des rythmes d'évolution. Géomorphologie : relief, processus, environnement, 8, 107-118.

COSTA S., DELAHAYE D., 2005 : Plages A Risques/Beaches At Risk (PAR/BAR). Rapport final. Conseil Régional de Haute-Normandie. Programme Européen Interreg IIIa, 175 pages.

COSTA S., LEVOY F., MONFORT O., CUROY J., DE SAINT LEGER E., DELAHAYE D., 2008 : Impact of sand content and cross-shore transport on the morphodynamics of macrotidal gravel beaches (Haute-Normandie, English Channel). Zeitschrift für Geomorphology, 3, 41-62.

DELLA-MARTA P. M., PINTO J. G., 2009 : Statistical uncertainty of changes in winter storms over the North Atlantic and Europe in an ensemble of transient climate simulations. Geophysical Research Letters, 36, L14703. doi:10.1029/2009GL038557.

ELINEAU S., DUPERRET A., MALLET P., CASPAR R., 2011 : Urban tidal flooding in Normandy (Le Havre, NW France). Geophysical Research Abstracts, 13, 201.

ESCOURROU G., 1978 : Climats et types de temps en Normandie. Thèse de Géographie, Université de Paris IV, 662 pages.

FLOHN H., 1968 : Le temps et le climat. Paris, Ed. Hachette, 253 pages.

GALLI F.L., HONTARREDE M., 2001 : Surcote et marée de tempête. Met. Mar., 49, 79-89.

HALLEGOUET B., HENAFF A., 2006 : Evolution récente et gestion des espaces littoraux de l'ouest Cornouaille. "Quelles pistes de développement pour le territoire ? "Actes des rencontres de l'ouest Cornouaille, 20-34.

HERRMANN T. M., PLANCHON O., 1998 : Climat et aménagement littoral en Poméranie occidentale (Allemagne/mer Baltique). Les publications de l'AIC, 11, 99-106.

JENNINGS R., SHULMEISTER J., 2002 : A field based classification scheme for gravel beaches. Marine Geology, 186, 211-228.

JOLY A., 1995. Le front polaire : un concept dépassé qui a la vie dure. La Recherche, 273, $128-135$. 
KEIM B. D., MULLER R. A., STONE G. W., 2004 : Spatial and temporal variability of coastal storms in the North Atlantic Basin. Marine Geology, 210, 7-15.

LAMBERT S., FYFE J. C., 2006 : Changes in winter cyclone frequencies and strengths simulated in enhanced greenhouse gas experiments: Results from the models participating in the IPCC diagnostic exercise. Climate Dynamics, 26, 713-728.

LECKEBUSCH G. C., KOFFI B., ULBRICH U., PINTO J.G., SPANGEHL T., ZACHARIAS S., 2006 : Analysis of frequency and intensity of winter storm events in Europe on synoptic and regional scales from a multi-model perspective. Climate Research, 31, 59-74.

LE CORNEC E., SCHOORENS J., 2007 : Etude de l'aléa submersion marine sur le site de la Grande Plage de Gâvres. Rapport d'étude DHI et GEOS, 103 pages.

LEROY M., 1999 : Classification d'un site. Notes techniques, DSO, Météo France, 35, 12 pages.

LEROY M., LAFAYSSE C., 1993 : Recommandations pour l'implantation et l'aménagement des parcs météorologiques. Notes techniques, DSO, Météo France, 31, 10 pages.

LOEPTIEN U., ZOLINA O., GULEV S., LATIF M., SOLOVIOV V., 2008 : Cyclone life cycle characteristics over the Northern Hemisphere in coupled GCMs. Climate Dynamics, 31, 507-532.

LOZANO I., DEVOY R.J.N., MAY W., ANDERSEN U., 2004 : Storminess and vulnerability along the Atlantic coastlines of Europe: Analysis of storm records and of a greenhouse gases induced climate scenario. Marine Geology, 210, 205-225.

MASON T., VOULGARIS G., SIMMONDS D.J., COLLINS M.B., 1997 : Hydrodynamics and sediment transport on composite (mixed sand/shingle) and sand beaches: A comparison. Coastal Dynamics, 48-57.

MASON T., COATES T.T., 2001 : Sediment transport processes on mixed beaches: A review for shoreline management. Journal of Coastal Research, 17, 645-657.

MASPATAUD A., 2011 : Impacts des tempêtes sur la morphodynamique du profil côtier en milieu macrotidal. Thèse de Géosciences, Géologie, Paléontologie, Océanographie, Université du Littoral Côte d'Opale, 470 pages.

MEEHL G. A., STOCKER T. F., COLLINS W. D., FRIEDLINGSTEIN P., GAYE A. T., GREGORY J. M., KITOH A., KNUTTI R., MURPHY J. M., NODA A., RAPER S. C. B., WATTERSON I. G., WEAVER A. J., ZHAO Z. C., 2007 : Global climate projections. In: SOLOMON S. et al. (eds), Climate change 2007: The physical science basis. Contribution of working group I to the fourth assessment report of the intergovernmental panel on climate change. Cambridge, UK and New-York, USA, Cambridge University Press, 747-845.

Météo-France, isofronts ( 06 h, 12 h, 18 h UTC).

Météo hebdo : Bulletin hebdomadaire d'études et de renseignements (12 h UTC).

MOREL V., 1997 : De la géomorphologie à la gestion des accumulations de galets littoraux du bassin de la Manche et de ses abords : études de cas (Bretagne, Haute-Normandie, Picardie, Sud Angleterre) et réflexions générales. Thèse de Géographie, Université de Brest, 286 pages.

ORFORD J. D., 1977 : A proposed mechanism for storm beach sedimentation. Earth Surface Processes, 2, 381-400. 
ORFORD J. D., JENNINGS S. C., FORBES D. L., 2001 : Origin, development, reworking and breakdown of gravel-dominated coastal barriers in Atlantic Canada: Future scenarios for the British coast. In: Packham J.R. (eds), British Shingles. Otley, Westbury, 23-55.

ORFORD J. D., FORBES D. L., JENNINGS S. C., 2002 : Organisational controls, typologies and time scales of paraglacial gravel-dominated coastal systems. Geomorphology, 48, 51-85.

PASKOFF R., 1985 : Les littoraux : impact des aménagements sur leur évolution. Paris, Ed. Masson, 188 pages.

PEETERS P., SCHOORENS J., LE CORNEC E., MICHARD B., LECHAT M., 2009 : Définition de l'aléa submersion marine sur le site de la Grande Plage de Gâvres (Morbihan). La Houille Blanche, 1, 45-51.

PERHERIN C., AZZAM C., KERGADALLAN X., 2012 : Révision du guide méthodologique plans de prévention des risques littoraux : une meilleure prise en compte de l'ensemble des phénomènes pour la connaissance de l'aléa submersion marine. Congrès $S H F:$ "Evènements extrêmes fluviaux et maritimes », Paris.

PIRAZZOLI P. A., COSTA S., DORNBUSH U., 2007 : Flood threat anomaly for the low coastal areas of the English Channel based on analysis of recent characteristic flood occurrences. Ocean Dynamics, 57, 501-510.

PIRAZZOLI P. A., COSTA S., DORNBUSH U., TOMASIN A., 2006 : Recent evolution of surge-related events and assessment of coastal-flooding risk on the eastern coasts of the English Channel. Ocean Dynamics, 56, 498-512.

PONTEE N. I., PYE K., BLOTT S., 2004 : Morphodynamic and sedimentary variation of mixed sand and gravel beaches, Suffolk, UK. Journal of Coastal Research, 1, 256-276.

SABATIER F., ANTHONY E. J., HEQUETTE A., SUANEZ S., MUSEREAU J., RUZ M. H., REGNAULT H., 2009: Morphodynamics of beach/dune systems: Examples from the coast of France. Géomorphologie : relief, processus, environnement, 1, 3-22.

SAVOURET E., 2007: Le concept d'événement géographique. Approche et réflexions à partir de l'étude environnementale et sociétale des impacts littoraux induits du Débarquement de Normandie du 6 juin 1944. Thèse de Géographie, Université de Caen Basse-Normandie, 360 pages.

SCAMPEI, 2011 : Scénarios Climatiques Adaptés aux zones de Montagne : Phénomènes extrêmes, Enneigement et Incertitudes. Site internet : http://www.cnrm.meteo.fr/scampei/

SIRACEDPC, 2008 : Plan ORSEC. Préfecture de Seine-Maritime. Document interne.

Site internet d'Infoclimat, réanalyse NCEP (00 h, 12 h UTC) : http://www.infoclimat.fr/modeles/ncep.php

Site internet de la NOAA : http://www.noaa.gov

Site internet de la Wetterzentrale, UKMO (00 h UTC) : http://www.wetterzentrale.de/topkarten/tkfaxbraar.htm

Site intemet de Météociel, réanalyseNCEP(00h, 12 hUTC): http:/www.meteociel.fr/modeles/archives/archives.php

Site internet de Météo-France sur les mécanismes et l'origine des tempêtes : http://www.cnrm.meteo.fr/dbfastex/recyf_temp/tempet004.html\#railzonal ; et .../tempet003.html\#railbloc

STEPHAN P., 2008 : Les flèches de galets de Bretagne : morphodynamiques passée, présente et prévisible. Thèse de Géographie, Université de Bretagne Occidentale, 560 pages.

SUANEZ S., 2009 : La question du bilan sédimentaire des côtes d'accumulation. Rôle des forçages naturels et anthropiques dans les processus morphodynamiques analysés à partir de quelques exemples pris en Méditerranée et en Bretagne. Habilitation à Diriger les Recherches, Université de Caen Basse-Normandie, 212 pages. 
SUANEZ S., FICHAUT B., SPARFEL L., 2007 : Méthode d'évaluation du risque de submersion des côtes basses appliquée à la plage du Vougot, Guissény (Bretagne). Géomorphologie : relief, processus et environnement, 4, 319-334.

SUANEZ S., CARIOLET J.-M., 2010 : L'action des tempêtes sur l'érosion des dunes, les enseignements de la tempête du 10 mars 2008. Norois, 215, 77-99.

TONISSON H., ORVIKU K., JAAGUS J., SUURSAAR U., KONT A, RIVIS R., 2008 : Coastal damages on Saaremaa Island, Estonia, caused by the extreme storm and flooding on January 9, 2005. Journal of Coastal Research, 24, 602-614.

TONNERRE M.-A., 2001 : Contributions à l'étude des tempêtes dans la Manche et en façade atlantique de la France, au nord de l'île de Noirmoutier, 1965-1994. Doctorat d'Etat, Université de Lille, 363 pages.

TRZPIT J.-P., 1977 : Les tempêtes nord-atlantiques : essai d'analyse géographique. Norois, 93, 33-52, et 94, 211-234.

TRZPIT J.-P., 1990 : Vivre avec les tempêtes dans l'Europe du Nord-Ouest. De l'évènement météorologique à la réalité vécue, Bulletin de la Société languedocienne de Géographie, 1-2, 164-202.

ULBRICH U., LECKEBUSCH G. C., PINTO J. G., 2009 : Extra-tropical cyclones in the present and future climate: A review. Theoretical and Applied Climatology, 96, 117-131.

VASSEUR B., HEQUETTE A., 2000 : Storm surges and erosion coastal dunes between 1957 and 1988 near Dunkerque (France), Southwestern North Sea. In: Pye K., Allen J.R.L. (eds), Coastal and Estuarine Environments. London, Geological Society, Special Publications, 175, 99-107.

WANG X. L., WAN H., ZWIERS F. W., SWAIL V. R., COMPO G. P., ALLAN R. J., VOSE R. S., JOURDAIN S., YIN X., 2011 : Trends and low-frequency variability of storminess over western Europe, 1878-2007. Climate Dynamics, 37, 2355-2371.

WOTH K., WEISSE R., VON STORCH H., 2006 : Climate change and North Sea storm surge extremes: an ensemble study of storm surge extremes expected in a changed climate projected by four different regional climate models. Ocean Dynamics, 56, 3-15. 